\title{
Determinanten des Online-Einkaufs - eine empirische Studie in sechs nordrhein-westfälischen Stadtregionen
}

\author{
Claus-C. Wiegandt ${ }^{7}$ Sabine Baumgart ${ }^{1}$. Nina Hangebruch ${ }^{1} \cdot$ Linus Holtermann $^{2} \cdot$ Christian Krajewski $^{3}$. \\ Matthias Mensing ${ }^{4} \cdot$ Cordula Neiberger $^{4} \cdot$ Frank Osterhage $^{5} \cdot$ Verena Texier-Ast $^{6} \cdot$ Klaus Zehner $^{8} \cdot$ Björn Zucknik $^{2}$
}

Eingegangen: 28. August 2017 / Angenommen: 28. März 2018 / Online publiziert: 17. April 2018

๑) Springer-Verlag GmbH Deutschland, ein Teil von Springer Nature 2018

\section{Zusammenfassung}

Einzelhandel findet heute nicht nur in den Stadt- und Stadtteilzentren bzw. in den Einkaufszentren am Rande der Städte statt, sondern zunehmend auch im Internet. Der Beitrag untersucht, welche Determinanten ausschlaggebend sind, im stationären Einzelhandel bzw. im Online-Handel einzukaufen. Die Ergebnisse basieren auf einer Befragung von rund 2.900 Personen in den sechs nordrhein-westfälischen Stadtregionen Aachen, Bochum, Bonn, Dortmund, Münster und Köln. In Abhängigkeit der Entfernung zur jeweiligen Innenstadt wurde in insgesamt 26 Untersuchungsgebieten befragt. In allen sechs Stadtregionen zeigt sich, dass nicht die räumlichen, sondern ausgewählte demographische und sozioökonomische Faktoren das Einkaufsverhalten bestimmen. Das Geschlecht, das Alter und die Lebensstile spielen beim Online-Einkauf eine wesentliche Rolle, das Einkommen ist keine beeinflussende Größe.

Schlüsselwörter Einzelhandel · Online-Handel · Einkaufsverhalten · Nordrhein-Westfalen

\section{Determinants of online shopping - an empirical study in six North Rhine-Westphalian city regions}

\begin{abstract}
Retail trade is no longer occurring in urban centres or suburban malls on the edge of cities, exclusively. It increasingly takes place online. The aim of this study is to examine the determining factors for in-store and online shopping respectively. The results are based on a survey of approximately 2,900 people in the six North Rhine-Westphalian city regions of Aachen, Bochum, Bonn, Dortmund, Münster and Cologne. Depending on the distance to each particular urban centre, 26 neighbourhoods were chosen to conduct the questionnaires. In all six city regions it is apparent that instead of spatial factors selected demographic and socio-economic forces determine buyer behaviour. While gender, age and lifestyles are important in terms of online shopping, income is no longer an influencing parameter.
\end{abstract}

Keywords Retail trade $\cdot$ E-commerce $\cdot$ Online shopping $\cdot$ Buyer behavior $\cdot$ North Rhine-Westphalia

\section{Einleitung}

Der Online-Handel hat in den vergangenen 20 Jahren die Einzelhandelsstrukturen in Deutschland schleichend und nachhaltig, vermutlich sogar irreversibel verändert. Dies hat weitreichende Folgen für die Angebots- und Nachfrageseite. Auf der Angebotsseite beeinflusst der Online-Handel die

Prof. Dr Claus-C. Wiegandt wiegandt@uni-bonn.de

Extended author information available on the last page of the article
Zusammensetzung und Umsatzentwicklung des stationären Einzelhandels in den Innenstädten und Stadtteilzentren. Dies führt dazu, dass der Online-Handel die Leistungsfähigkeit der Zentren beeinträchtigen kann - in einigen Fällen mehr, in anderen Fällen weniger. Zudem kommt es durch den Online-Handel zu neuen Anforderungen an die Logistik. Die Ausweitung des Lieferverkehrs und die an einigen Stadträndern entstehenden Logistikzentren bleiben nicht ohne Folgen für den Stadtverkehr und das Siedlungsbild. Diese Themen auf der Angebotsseite werden bereits in den überregionalen Tageszeitungen (Kläsgen 2017; Zacharakis 2017), auf Fachtagungen, in wissenschaftlichen Abschlussarbeiten (Langsenkamp 2014; Stepper 2015) und 
Studienprojekten sowie in Gutachten (KPMG 2016; BMWi 2017) und politischen Positionspapieren (ZIA 2015; DST 2016) intensiv diskutiert.

Veränderungen durch den Online-Handel gibt es aber auch auf der Nachfrageseite. Hier stellt sich die Frage, welche Determinanten für das Einkaufsverhalten der Konsumenten im stationären Einzelhandel in klassischer Form bzw. im Online-Einkauf in digitaler Form ausschlaggebend sind. Dabei ist zwischen Faktoren, die sich aus den demographischen bzw. sozioökonomischen Dispositionen der Konsumenten ergeben, und räumlichen Faktoren, die aus den Angeboten im stationären Einzelhandel vor Ort bzw. der Herkunft der Konsumenten resultieren, zu unterscheiden. Es stellt sich zudem die Frage, ob es bei den Konsumenten Unterschiede im Einkaufsverhalten zwischen verschiedenen Städten gibt. Zentrales Erkenntnisinteresse des Beitrages ist es, in welchem Verhältnis diese einzelnen Determinanten des Einkaufsverhaltens zueinander stehen.

Somit sollen folgende Forschungsfragen im Rahmen einer eigenen empirischen Untersuchung bearbeitet werden:

- Welche Motive beeinflussen das Einkaufsverhalten im stationären Einzelhandel bzw. im Online-Handel? (Kapitel 5)

- Welchen Einfluss haben demographische und sozioökonomische Faktoren für das Einkaufsverhalten im stationären Einzelhandel bzw. im Online-Handel? (Kapitel 6)

- Inwiefern sind verschiedene Lebensstiltypen und damit verbunden unterschiedliche Affinitäten zur Nutzung des Internets bestimmend für die Wahrnehmung von Angeboten im stationären Einzelhandel oder im Online-Handel? (Kapitel 6)

- Inwiefern beeinflusst die Attraktivität der Innenstädte und Stadtteilzentren das Einkaufsverhalten der Konsumenten bezogen auf den stationären Einzelhandel vor Ort bzw. den Online-Handel? (Kapitel 7)

- Welche Rolle spielt die Entfernung zwischen dem Wohnort der Konsumenten und den Einzelhandelsstandorten in den Innenstädten für das Einkaufsverhalten im stationären Einzelhandel bzw. im Online-Handel? (Kapitel 7)

Mit der Beantwortung dieser Forschungsfragen werden neue empirische Erkenntnisse zu den Determinanten des Einkaufsverhaltens präsentiert, die mit ihren räumlichen Bezügen der kommunalen Planungspraxis, Unternehmen und Investoren im Einzelhandel sowie der weiteren wissenschaftlichen Beschäftigung mit diesem Thema dienen können. Aufbauend auf den eigenen empirischen Erkenntnissen werden deshalb im achten Kapitel Handlungsempfehlungen für die Stadtentwicklungspolitik in Deutschland formuliert. Damit trägt der Beitrag der Empfehlung der Bundesregierung Rechnung, Forschungsergebnisse als Wissenstransfer zu generieren und zu veröffentlichen (BMWi 2017: 33). Der Anspruch ist hingegen nicht, den Zusammenhang von
Einkaufsverhalten und seinen Determinanten theoretischkonzeptionell weiterzuentwickeln. Nach einem einführenden Überblick zum Online-Handel in Deutschland im zweiten Kapitel wird im dritten Kapitel des Beitrags der Forschungsstand zum Online-Handel kurz vorgestellt, um darauf aufbauend im vierten Kapitel das Forschungsdesign zu präsentieren. Im fünften, sechsten und siebten Kapitel werden die Ergebnisse der quantitativen Haushaltsbefragung in sechs nordrhein-westfälischen Stadtregionen dargelegt. Darauf basierend werden zum Abschluss einige Schlussfolgerungen für zukünftige Handlungsansätze der räumlichen Planung gezogen.

\section{Entwicklung des Online-Handels in Deutschland}

Der Einzelhandel in Deutschland unterliegt seit Jahrzehnten einem kontinuierlichen Strukturwandel, der sowohl auf Veränderungen der Nachfrageseite (gesellschaftlicher Wandel) als auch auf Veränderungen der Angebotsseite (Unternehmenskonzentration und Betriebsformenwandel) zurückzuführen ist (BMWi 2017: 3; Kulke 2017: $171 \mathrm{ff}$.). Dieser Strukturwandel bedingt auch räumliche Selektionsprozesse. So löst sich etwa das feinmaschige Netz kleinflächiger Betriebsformen im Lebensmitteleinzelhandel auf und es entsteht ein wesentlich grobmaschigeres Netz großflächiger Betriebsformen. Dadurch kann die Nahversorgung in vielen Kommunen nicht mehr flächendeckend (oder auch gar nicht) aufrechterhalten werden (Jürgens 2014). Auch in den Non-Food-Warengruppen konzentrieren sich die Umsätze auf großflächige Betriebsformen der Filialisten, die sich in den größeren Städten und wenigen 1a-Lagen sowie in mehr oder weniger gut integrierten Shopping-Centern finden. So geraten Stadtteilzentren, kleinere Städte und Mittelstädte unter Druck und zunehmend in Prozesse der Gebietsabwertung (Trading Down), die für einige Einkaufslagen bis hin zu ganzen Innenstädten und Stadtteilzentren erhebliche Leerstände und damit auch einen Verlust für Ortsbildund Aufenthaltsqualitäten mit sich bringen (Pesch/Schenk/ Sperle 2003).

Verschärft wird diese Situation seit Anfang der 2000erJahre durch die Verlagerung von Kaufkraft in den Online-Handel. Über diesen Vertriebsweg sind - nach jeweils jährlichen zweistelligen Wachstumsraten - im Jahr 2016 rund $10 \%$ aller Einzelhandelsumsätze in Deutschland abgewickelt worden (HDE 2017: 3). Die Anteile des OnlineHandels differieren sehr stark nach Warengruppen sowie im Zeitablauf. Da insbesondere in den typischerweise innenstadt- bzw. zentrenrelevanten Sortimentsgruppen bereits frühzeitig eine Umsatzverlagerung in den Online-Handel erfolgte, ist eine Auseinandersetzung mit diesen Phänomenen sowohl für die Stadtforschung als auch die Stadt- 
entwicklungsplanung von Belang. So wurden im Bereich „Technik und Medien“ (z. B. Elektrik, Elektronik, Bücher) schon sehr früh Waren online vertrieben und gekauft. Zwischen 2011 und 2013 erlangte diese Gruppe ein jährliches Umsatzwachstum von 20 bis $30 \% .2014$ bildeten diese Sortimente mit einem Online-Anteil von $20,9 \%$ am Gesamthandelsvolumen auch die stärkste Warengruppe im OnlineHandel. Allerdings ist mittlerweile eine Abschwächung des jährlichen Wachstums auf etwa 8 \% (2013 auf 2014) zu verzeichnen, im Bereich „Bücher und Schreibwaren“ sogar auf nur noch $2,4 \%$. Andere Warengruppen folgten dieser Entwicklung, wie „Fashion und Lifestyle“, die heute einen Online-Anteil von 18,9\% aufweist, allerdings mit rückläufigen Wachstumsraten (GfK Geo Marketing 2015: 6). Getrieben wird die Gesamtzunahme des Online-Anteils heute durch sogenannte Nachzüglerbranchen, die nun ein überdurchschnittliches Wachstum erkennen lassen, wie „Wohnen und Einrichten“, „Heimwerken und Garten“ und „FMCG“ (Fast Moving Consumer Goods, also Lebensmittel und Drogeriewaren) (HDE 2017: 9).

So wird heute das moderate Wachstum des gesamten Einzelhandelsumsatzes (12\% seit 2000) zu einem Großteil durch den Online-Handel generiert (HDE 2017: 9). Ein stagnierendes Bevölkerungswachstum, ein verändertes Ausgabeverhalten (weniger Konsumartikel, mehr Dienstleistungen) sowie nur leichte Kaufkraftzunahmen lassen für die Zukunft zudem nicht auf einen Umsatzzuwachs im stationären Handel hoffen. Im Gegenteil gehen Prognosen von einem weiteren Wachstum des Online-Handels aus, der zunehmend Kaufkraft aus dem stationären Handel abschöpfen wird. Damit wird der Online-Handel den Strukturwandel des Einzelhandels auch in Zukunft als Trendverstärker vorantreiben (BBSR 2017: 29).

Allerdings wird nicht davon ausgegangen, dass das Wachstum des Online-Handels in den nächsten Jahren in der gleichen Dynamik weiterverlaufen wird. Vielmehr wird prognostiziert, dass es bei einem durchschnittlichen Wachstum des Online-Handels von etwa $10 \%$ bei gleichzeitigem Wachstum des gesamten Einzelhandelsumsatzes von etwa einem Prozent pro Jahr zu einem Online-Anteil von etwa $15,7 \%$ kommen wird. Bei wiederum halbierter Wachstumsrate bis 2025 wäre von einem Online-Umsatz von dann 98,2 Mrd. Euro bei einem gesamten Einzelhandelsumsatz von $508 \mathrm{Mrd}$. Euro auszugehen, was einem Anteil von 19,3\% entsprechen würde (BBSR 2017: 35 f.).

Online-Handel und stationärer (Offline-)Handel stehen jedoch nicht mehr in einem konträren Verhältnis. Heute und in Zukunft werden Online-Umsätze nicht nur von reinen Online-Händlern (Internet Pure Player) generiert, sondern auch von Online-Shops, die durch stationäre Händler, Hersteller oder Versandhändler betrieben werden. Kunden machen zunehmend keinen Unterschied mehr zwischen den einzelnen Vertriebsformen, sondern agieren warengruppen- und situationsbedingt (vgl. Heinemann 2014). Besonders beliebt ist dabei die Kombination aus Ladengeschäft und Online-Shop, da hier zwischen den Vertriebskanälen gewechselt werden kann bzw. diese kombiniert werden können, beispielsweise durch Online-Recherche und Bestellung der Ware ins Geschäft, um diese dann dort anzusehen und gegebenenfalls auszuprobieren. Ebenso gibt es die Möglichkeit einer Bestellung nach Hause, aber einer Rückgabe der Ware im Geschäft. Zudem werden weitere Formen der Verknüpfung von stationärem Einzelhandel und OnlineHandel praktiziert, z. B. über Augmented Reality (AR), das heißt die digitale Erweiterung der Realität, etwa bei der virtuellen Anprobe von Kleidungsstücken (KPMG 2016: 24). Dieses Cross Channeling bieten insbesondere große Einzelhändler an. So gewinnen Online-Shops der stationären Händler zurzeit Anteile am gesamten Online-Umsatz (IFH 2016: 8). Online-Umsätze können somit durchaus eine stationäre Präsenz des Einzelhandels stützen. Ebenso ist in jüngerer Zeit zu beobachten, dass auch reine Internet-Händler Ladengeschäfte eröffnen, um ihre Bekanntheit zu steigern (vgl. Hover 2016). Damit verschwimmen die Grenzen zwischen Online- und Offline-Handel weiter. Die Handelsunternehmen setzen also verstärkt auf Mehrkanal-Handel (Multi Channel). Dabei ist zu berücksichtigen, dass die Kombination verschiedener Angebotskanäle für den Handel mit einem deutlich erhöhten Aufwand einhergeht, der insbesondere für viele kleinere Unternehmen nur schwer leistbar ist.

\section{Stand der Forschung und Erkenntnisse über Determinanten des Einkaufsverhaltens}

Um das Wachstum des Online-Handels zu analysieren, kommt dem Verständnis des Kundenverhaltens eine wichtige Rolle zu. Wissenschaftliche Beiträge weltweit beziehen sich daher in erster Linie auf die Frage von Schlüsselfaktoren, die das Konsumentenverhalten im Online-Handel beeinflussen. Dies sind neben Produkteigenschaften und Eigenschaften der Vertriebskanäle insbesondere Einstellungen und Wahrnehmungen sowie demographische und sozioökonomische Merkmale der Konsumenten. Bisher haben nur wenige Studien ihre Aufmerksamkeit räumlichen Determinanten gewidmet (vgl. Mensing/Neiberger 2016).

Eine Übersicht über bisherige empirische Ergebnisse fällt insofern schwer, als dass sich die Studien auf unterschiedliche Untersuchungsräume, Stichprobengrößen und -zusammensetzungen, Methoden und Erhebungszeiträume beziehen. So ist es nicht verwunderlich, dass die internationalen Forschungsergebnisse zum Teil durchaus widersprüchlich erscheinen. Einige Befunde lassen sich dennoch als weitgehend anerkannt herauskristallisieren: 
- Von großer Bedeutung sind die Erfahrungen der Kunden im Umgang mit dem Computer und dem Internet. Verschiedene Autoren haben gezeigt, dass eine häufige Nutzung der neuen Technologien die Wahrscheinlichkeit des Online-Einkaufs erhöht (vgl. Dijst/Farag/Schwanen 2008, Kacen/Hess/Wei-Yu 2013).

- Einstellungsmerkmale, wie die Motivation, die Intention, die Zufriedenheit oder der Lebensstil der Kunden spielen eine maßgebliche Rolle bei ihren Entscheidungen, stationär oder via Internet einzukaufen. So sind die Möglichkeiten, freie und jederzeit zugängliche Produktinformationen sowie Produkte günstiger als im stationären Geschäft über das Internet zu erhalten, eine Motivationsquelle zur Beschaffung von Gütern über das Internet. Im Gegensatz dazu reduzieren ein zu geringes Sicherheitsgefühl oder eine potenziell gefährdete Privatsphäre bei der Bezahlung das Vertrauen in den Verkaufskanal und somit die Intention, über das Internet einzukaufen (vgl. Burroughs/Sabherwal 2002; Gefen/Karahanna/Straub 2003; Swinyard/Smith 2003).

- Eine große Anzahl von Studien findet einen Zusammenhang zwischen dem Alter der Kunden und der Nutzung des Online-Handels. In diesen Fällen kaufen tendenziell jüngere Kunden häufiger im Internet ein (Wu 2003; Sorce/Perotti/Widrick 2005; Brashear/Kashyap/Musante et al. 2008; Passyn/Diriker/Settle 2011; Gong/Stump/ Maddox 2013). Des Weiteren beschreiben vor allem ältere Studien, dass im Verhältnis eher männliche Personen affin gegenüber dem Online-Einkauf sind und dementsprechend häufiger diesen Verkaufskanal nutzen als Frauen (Farag/Dijst/Lanzendorf 2003; Girard/ Korgaonkar/Silverblatt 2003). Neuere Studien finden den Zusammenhang allerdings nicht mehr (Hernández/Jiménez/Martín 2011). Bildung, Einkommen und sicherer Umgung mit Computern beeinflussen in positiver Richtung die Nutzung des Online-Handels. Je besser ein Konsument gebildet ist bzw. je höher sein Bildungsabschluss ist, desto wahrscheinlicher ist die Nutzung des Internets zur Beschaffung von Gütern (Girard/ Korgaonkar/Silverblatt 2003; Farag/Krizek/Dijst 2006; Weltevreden/van Rietbergen 2009; Hernández/Jiménez/ Martín 2011; Lian/Yen 2014).

- Die Ausstattung des Raumes mit Informations- und Kommunikationsinfrastruktur hat einen Einfluss auf die Nutzung des Online-Handels (Cao/Douma/Cleaveland 2010; Nerlich/Schiffner/Vogt 2010).

Die Frage nach der Bedeutung räumlicher Determinanten, wie die Ausstattung mit Geschäften sowie die Entfernung zur nächsten Einkaufsmöglichkeit bzw. zum nächsten Stadtzentrum, konnte bisher nicht eindeutig beantwortet werden. So haben die Autoren einer niederländischen Studie festgestellt, dass die Intention, Elektronikprodukte im Internet zu bestellen, reduziert ist, wenn sich in der Nähe der Kunden eine höhere Anzahl an Elektronikmärkten befindent (Dijst/Farag/Schwanen 2008: 842f.). Dass Einkaufsmöglichkeiten in der Nähe der Kunden die Internetaktivität reduziert, stellten auch Ren und Kwan (2009) fest. Allerdings weisen sie darauf hin, dass es sich dabei nur um einen sehr geringen Effekt handelt (Ren/Kwan 2009: 276).

Eine Gegenthese zur Bedeutung räumlicher Determinanten besagt, dass die festzustellenden räumlichen Unterschiede im Online-Kaufverhalten auf die unterschiedliche soziodemographische Zusammensetzung der Bevölkerung in verschiedenen Raumkategorien zurückzuführen sind. Diese wurde von Farag, Krizek und Dijst (2006) für die Niederlande bestätigt. Die Autoren führen zwar aus, dass umso mehr online eingekauft wird, je kürzer die Distanz zu den Einkaufsstätten ist. Sie begründen dies allerdings mit der räumlichen Segregation der Bevölkerung. Auch Sinai und Waldfogel (2004) gehen davon aus, dass in den USA Internet und Online-Kauf in größeren Städten eine bedeutendere Rolle spielten als in kleineren. Sie finden aber ebenso Argumente für die Distanzabhängigkeit, da sie feststellten, dass mehr Geld für Online-Käufe von Büchern und Kleidung ausgegeben wird als für Offline-Käufe, wenn die Menschen weiter weg von den stationären Läden wohnen (Sinai/Waldfogel 2004: 22).

Mensing und Neiberger (2016) untersuchten das OnlineOffline-Kaufverhalten in sechs Kommunen in drei unterschiedlichen Raumkategorien (hochverdichtete Agglomerationsräume, verstädterte Räume mittlerer Dichte und ländliche Räume). Dabei wurde deutlich, dass das vorgefundene unterschiedliche räumliche Muster nicht auf die Ausstattung der Räume mit Ladengeschäften zurückgeführt werden kann, sondern auf die sozioökonomischen Merkmale der Bevölkerung.

Zusammenfassend lässt sich sagen, dass die Erkenntnisse aus den vielfältigen internationalen Studien hinsichtlich sozioökonomischer Aspekte und räumlicher Determinanten heterogen sind. Zu berücksichtigen sind zudem die unterschiedlichen Zeiträume ihrer Entstehung. Auffallend bei der Vielfalt der Beiträge ist, dass sich nur sehr wenige empirische Studien auf Deutschland beziehen, obwohl gerade hier eine starke Dynamik des Online-Handels festzustellen ist und schon frühzeitig erkannt wurde, dass diese Entwicklung eine Verschärfung des Strukturwandels und damit einhergehend eine Beschleunigung der Selektionsprozesse im stationären Einzelhandel mit sich bringen wird. Gerade im Hinblick auf die Frage nach den Wirkungen des zunehmenden Online-Handels auf die Städte als Agglomerationen des Einzelhandels lassen die zitierten Beiträge nur wenige Schlussfolgerungen zu. Vor diesem Hintergrund soll die hier vorliegende Studie neue Erkenntnisse liefern, indem einerseits eine umfassende empirische Basis geschaffen wird und andererseits ein weiterer, bisher 
nicht untersuchter Aspekt eingeführt wird: die Attraktivität der Innenstädte. Ansatzpunkt der Überlegung ist, dass die Verbraucher nicht immer rational nur die räumlich nächstgelegenen Einkaufsstandorte aufsuchen, sondern im Rahmen einer Mehrfachorientierung verschiedene Zentren je nach Einkaufsanlass und Produkt besuchen. Dabei werden auch als attraktiv eingeschätzte Standorte ausgewählt, die in weiterer Entfernung liegen (Gebhardt 2002). Wird der Online-Handel ebenso als konkurrierender „Standort“ betrachtet, lässt sich schlussfolgern, dass diejenigen, die den von ihnen bevorzugten Einkaufsstandort als besonders attraktiv einschätzen, auch weniger häufig online einkaufen. Vor diesem Hintergrund sind die Forschungsfragen formuliert (vgl. Kapitel 1).

\section{Methodisches Vorgehen: Umfrage in 26 Untersuchungsgebieten in sechs nordrhein-westfälischen Stadtregionen}

Dieser Beitrag möchte die im vorangegangenen Kapitel aufgezeigte Lücke in der empirischen Forschung zum Einkaufsverhalten schließen. Im Rahmen eines gemeinsamen Studienprojekts an sechs nordrhein-westfälischen Hochschulen wurde im Wintersemester 2016/2017 in sechs Städten (Aachen, Bonn, Bochum, Dortmund, Köln und Münster) und einigen ausgewählten Umlandgemeinden eine Umfrage mit einem Rücklauf von rund 2.900 Befragten durchgeführt und ausgewertet, um so Aussagen zu den bestimmenden Faktoren des Einkaufsverhaltens treffen und das Verhältnis von Online- zu Offline-Einkäufen klären zu können. Untersucht wurde auch dabei das Verhältnis von Einstellungen zur Attraktivität der Innenstädte und zu den Vor- und Nachteilen des Einkaufs im Online-Handel bzw. im stationären Einzelhandel, die demographischen und sozioökonomischen Merkmale der Befragten sowie räumliche Determinanten, um die zentralen Forschungsfragen zu klären (vgl. Kapitel 1). Die sechs untersuchten Stadtregionen repräsentieren sowohl prosperierende als auch schrumpfende Regionen.

Um vergleichbare Ergebnisse zum Einkaufsverhalten der Bevölkerung in den sechs Stadtregionen zu erhalten, wurde ein standardisierter Fragebogen entwickelt. Er umfasst Fragen

- zur Internetaffinität der Befragten,

- zum bevorzugten Ort des Einkaufs ausgewählter Produkte und zur Häufigkeit dieser Einkäufe online oder offline,

- zur Bewertung der Attraktivität der Einkaufsorte, insbesondere zum Angebot an Einzelhandelsgeschäften, zur Aufenthalts- und Verweilqualität und zur Erreichbarkeit der Innenstädte und Stadtteilzentren,
- zu Motiven für den Einkauf im klassischen Ladengeschäft bzw. im Internet und zum persönlichen Einkaufsverhalten,

- zu Lebensstilen und

- zu den demographischen und sozioökonomischen Merkmalen der Befragten.

Die Frage nach der Anzahl der Einkäufe im Ladengeschäft bzw. im Internet für ausgewählte Produktgruppen erstreckte sich über die letzten drei Monate vor der Befragung (September, Oktober, November 2016). Den Probanden wurde die Frage gestellt, wie oft sie bestimmte Produkte in den zurückliegenden drei Monaten einerseits im Laden und andererseits online gekauft haben. Bei den Produkten, nach denen gefragt wurde, handelte es sich um Elektroartikel, Bücher, Sportartikel, Bekleidung, Schuhe, Haushaltswaren, Drogerieartikel sowie Lebensmittel. Die Einteilung war an der Sortimentsgliederung orientiert, die der Landesentwicklungsplan Nordrhein-Westfalen verwendet (Staatskanzlei Nordrhein-Westfalen 2013). Der Zeitraum von drei Monaten wurde vorgegeben, weil dieser in der Regel von Käufern noch überblickt wird und somit realitätsnahe Antworten erwartet werden können. Zudem ist er so lang, dass das in ihm praktizierte Einkaufsverhalten als repräsentativ angenommen werden kann. Aus den Antworten konnte für jeden Probanden der Anteil der Online-Käufe zum einen über alle Produktgruppen summiert und zum anderen produktgruppenspezifisch errechnet werden (vgl. Kapitel 6).

Zur Erfassung der Lebensstile wurde auf die Typologie der Lebensführung nach Otte (2005) zurückgegriffen. Dieses Konzept der Lebensstiltypen dient zur Beantwortung der zentralen Frage, ob eher individuelle Charakteristika der Befragten (demographische und sozioökonomische Merkmale, Lebensstile) oder räumliche Komponenten (Attraktivität des Angebots in den Zentren, räumliche Distanz zum Angebot) über die Präferenz entscheiden, im stationären Einzelhandel oder online einzukaufen.

In den sechs Stadtregionen wurde in insgesamt 26 Untersuchungsräumen befragt (vgl. Abbildung 1). Die Auswahl erfolgte mit dem Ziel, eine möglichst große Heterogenität bei den Untersuchungsräumen hinsichtlich der Erreichbarkeit der jeweiligen Innenstädte zu erzielen. Drei Erreichbarkeitsklassen wurden über die Fahrzeit mit dem öffentlichen Personennahverkehr zur Innenstadt gebildet (weniger als 10 Minuten, 10 bis 30 Minuten, mehr als 30 Minuten). Danach wurden bis zu fünf Untersuchungsräume pro Stadtregion so ausgewählt, dass sich mindestens ein Untersuchungsraum in einer der drei Klassen befindet. Aus jedem Untersuchungsgebiet stammen rund 100 ausgefüllte Fragebögen.

Die zu befragenden Haushalte in den Stadtteilen und Quartieren - und bewusst nicht an den Standorten des Einzelhandels in den Innenstädten oder den Stadtteilzentren 

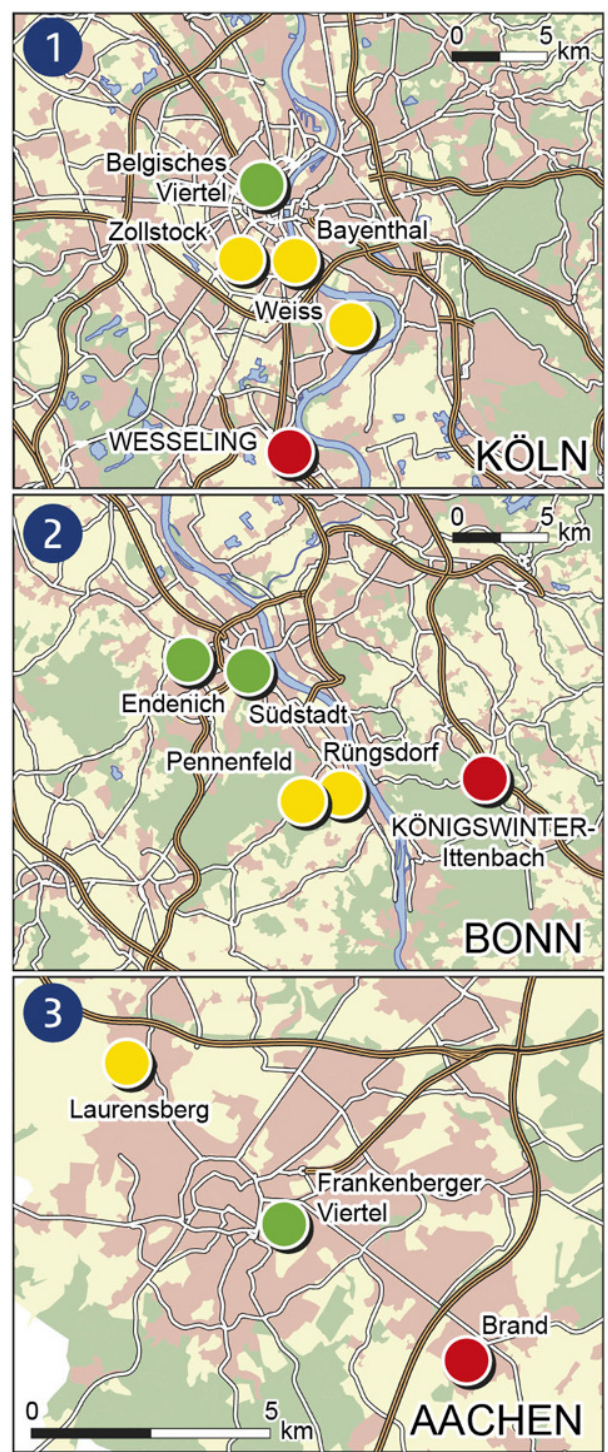
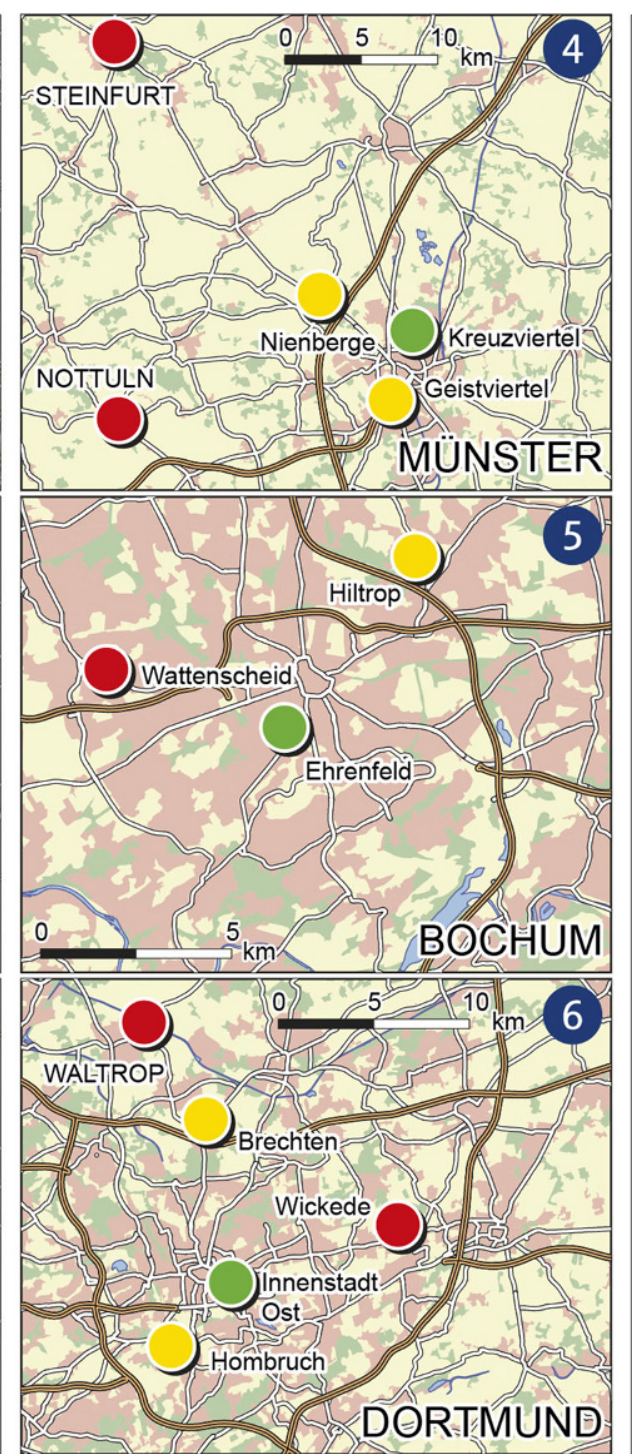

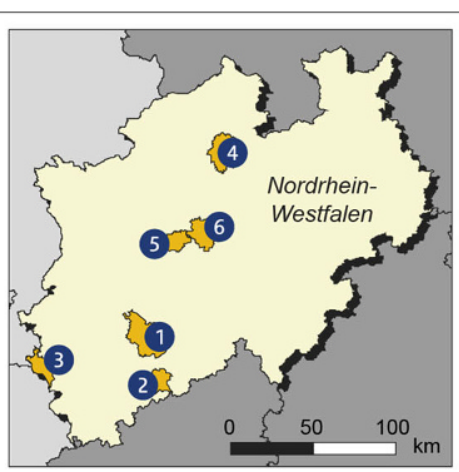

Erreichbarkeit der Innenstadt mit dem ÖPNV

Fahrzeit

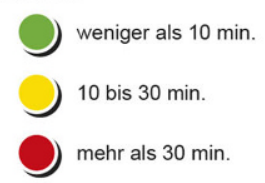

Abbildung 126 Untersuchungsgebiete in sechs Stadtregionen in Nordrhein-Westfalen (CORINE Land Cover CLC, 2012; GADM 2017; OSM 2017; Kartographie: U. Schwedler)

- wurden mithilfe eines zufallsgesteuerten Auswahlverfahrens bestimmt. Dazu wurden in der Regel zunächst einzelne Straßen in den Untersuchungsgebieten gewählt, die für das jeweilige Quartier repräsentativ sind. Dort wurde dann in jedem zweiten Haus befragt. Innerhalb der Häuser wurden die Probanden nach dem Zufallsprinzip ausgewählt. Die Befragung wurde in mehreren Stufen durchgeführt, um einen möglichst hohen Rücklauf zu generieren. In einem ersten Schritt wurden die Probanden vor Ort persönlich an ihren Wohnstätten befragt. Durch Ankündigungsschreiben und Presseinformationen waren die Haushalte auf die Umfrage vorbereitet worden. Konnten die ausgewählten Haushalte (wiederholt) nicht erreicht werden, wurden die Fragebögen in die Briefkästen eingeworfen und den Probanden wurde angeboten, die Befragung eigenständig durchzuführen. Dies konnte einerseits über eine Plattform im Internet erfolgen, die über einen Link bzw. einen QR-Code auf den Fragebögen erreicht werden konnte. Andererseits konnte der Bogen analog per Post zurückgesendet werden. Auch die Abholung des Fragebogens an einem benannten Termin war möglich. Als in einigen Untersuchungsgebieten absehbar war, dass der erwünschte Rücklauf von 100 Fragebögen nicht erreicht werden konnte, wurde zudem an öffentlichen und nichtöffentlichen Orten in den Quartieren selbst (z. B. an Plätzen, vor Supermärkten) befragt. Hier wurde darauf geachtet, dass die Befragten ihren Wohnsitz in den Quartieren hatten, um die Zugehörigkeit zur Zielgruppe sicherzustellen. Angesichts des Umfangs der Studie und der unterschiedlichen Stadtregionen und Untersuchungsgebiete wurde in Kauf genommen, dass die Befragungssituationen nicht völlig identisch waren. Insgesamt wurden 11.435 Haushalte in den 26 Untersuchungsgebieten gebeten, dass 
eine Person aus dem Haushalt an der Befragung teilnimmt. Auf diese Weise wurden 2.881 Fragebögen ausgefüllt. Mit einem Rücklauf von 25,2 \% konnte eine akzeptable und für wissenschaftliche Zwecke hinreichende Rücklaufquote erzielt werden.

\section{Individuelle Einstellungen und Kontextbedingungen als Determinanten des Einkaufsverhaltens}

Zunächst geht es in diesem Kapitel um die Einstellungen und Motive, die das Einkaufsverhalten im stationären Einzelhandel bzw. im Online-Handel beeinflussen. Im fünften, sechsten und siebten Kapitel werden die Forschungsfragen (vgl. Kapitel 1) beantwortet.

Um die Stärken des stationären Einzelhandels im Wettbewerb mit dem Online-Handel zu identifizieren, wurden die Probanden in allen sechs Stadtregionen auf der Basis von Antwortvorgaben nach den wichtigsten Gründen für den Einkauf im Ladengeschäft befragt. Von jeweils mehr als $80 \%$ der Interviewpartner werden die sofortige Mitnahme der gekauften Waren und deren Begutachtung als ausschlaggebende Kriterien für den Einkauf im Ladenlokal angesehen (vgl. Abbildung 2). Bedeutende Gründe für den Einkauf vor Ort stellen zudem die Unterstützung des lokalen Einzelhandels (65\%) sowie die Kopplung mit anderen Tätigkeiten dar (53\%). Datenschutzrechtliche Bedenken oder eine fehlende Technikkompetenz sind kaum relevante Motive für den Einkauf im Ladengeschäft.
Eine differenzierte Analyse der Gründe für den Einkauf im Ladengeschäft nach der Entfernung, dem Alter und dem Haushaltseinkommen führt zu folgenden Erkenntnissen: Zunächst lässt sich festhalten, dass Haushalte, für die die Innenstadt in weniger als zehn Minuten zu erreichen ist, ihren Einkauf deutlich häufiger mit weiteren Tätigkeiten koppeln als Haushalte, die weiter entfernt wohnen. Die Beratung vor Ort oder das Einkaufserlebnis ist für Haushalte dieses Entfernungstyps (unter 10 Minuten) weniger bedeutsam als für Haushalte, die in einem Quartier mit größerer Entfernung zur Innenstadt wohnen (10 bis 30 Minuten oder über 30 Minuten). Eine deutliche Alters- sowie Einkommensabhängigkeit lässt sich für die Beweggründe „Beratung vor Ort“ und „Unterstützung des lokalen Einzelhandels" feststellen, wobei die Bedeutung für die Alterskohorte 65 Jahre und älter sowie für die Gruppe mit einem Nettohaushaltseinkommen von über 4.500 Euro/Monat jeweils am höchsten ist. Im Vergleich zu höheren Einkommensklassen finden Personen mit geringerem Haushaltsnettoeinkommen (unter 2.000 Euro/Monat) die sofortige Mitnahme von Waren, die Kopplung mit anderen Tätigkeiten oder auch das Einkaufserlebnis wichtiger (vgl. Abbildung 3).

Als eine weitere Determinante wurde die Wirkung der Internet-Affinität auf das Online-Einkaufsverhalten der Befragten geprüft. Die Internet-Affinität wurde im Rahmen einer eigenen Frage erfasst, in der sich die Befragten $\mathrm{zu}$ sechs verschiedenen Aktionen im Internet äußern sollten. Wurden sämtliche Fragen nach Online-Aktionen mit „,nein“ beantwortet, wurde keine Internet-Affinität unterstellt. Wurde hingegen ein- oder zweimal mit ,ja“ geantwortet, wurde
Abbildung 2 Bedeutende Gründe für den Einkauf im Ladengeschäft

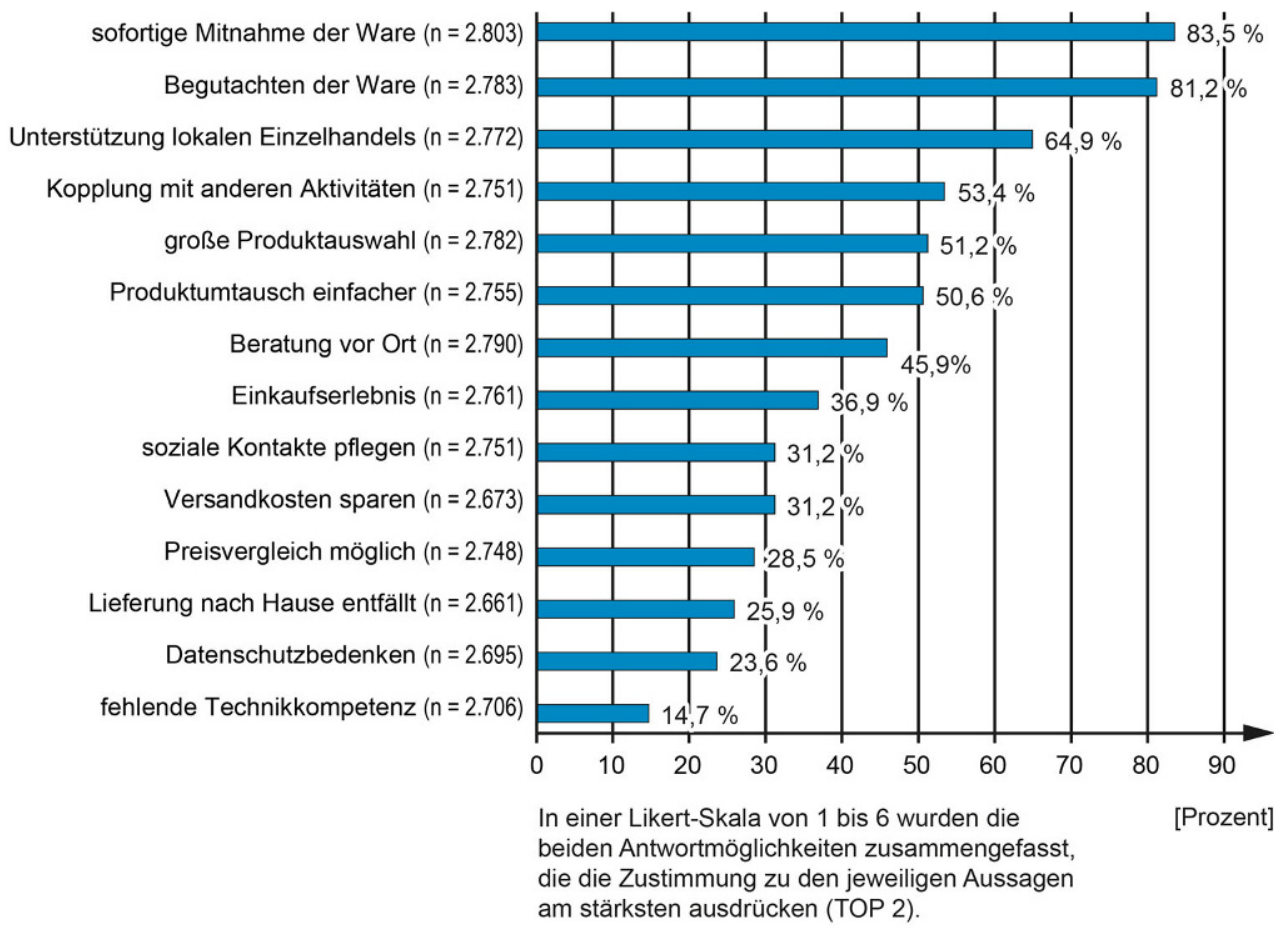

Quelle: eigene Erhebungen 2016 


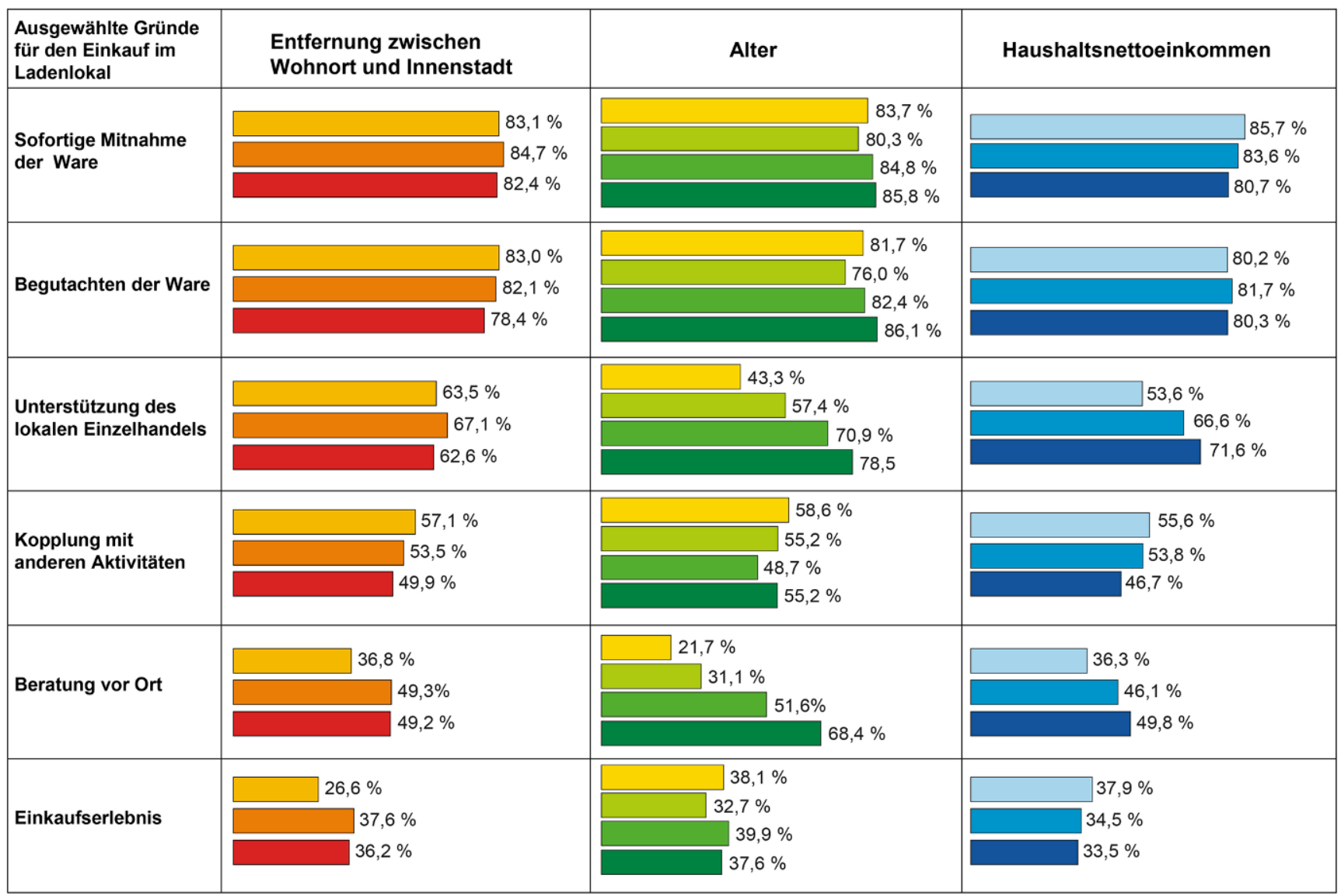

In einer Likert-Skala von 1 bis 6 wurden die beiden Antwortmöglichkeiten zusammengefasst, die die Zustimmung zu den jeweiligen Aussagen am stärksten ausdrücken (TOP 2).

unter 10 min. Entfernung $(n=776)$

10 bis $30 \mathrm{~min}$. Entfernung $(\mathrm{n}=1.192)$

über $30 \mathrm{~min}$. Entfernung $(n=893)$ unter 24 Jahre $(n=351)$

24 bis 44 Jahre $(n=793)$

45 bis 64 Jahre $(n=963)$

65 Jahre und älter $(n=641)$ unter $2000 €(n=539)$

2000 bis $4500 €(n=993)$

über $4500 €(n=540)$

Quelle: eigene Erhebungen 2016

Abbildung 3 Ausgewählte Gründe für den Einkauf im Ladengeschäft, differenziert nach Entfernungstypen, Alter und Nettoeinkommen des Haushalts

von einer schwach ausgeprägten Internet-Affinität ausgegangen. Entsprechend wurden drei bis fünf mit ,ja“ beantwortete Fragen als Indiz für eine stark ausgeprägte InternetAffinität gewertet. Wurden schließlich alle Fragen mit ,ja“ beantwortet, wurde die Internet-Affinität als sehr stark interpretiert. Die Aktionen, nach denen gefragt wurde, bezogen sich auf die Informationssuche, die Kommunikation über E-Mails, das Streamen von Filmen, die Nutzung sozialer Netzwerke, das Online-Banking und den Kauf von Tickets im Internet.

Die Differenzierung nach dem Merkmal Internet-Affinität der Befragten (zwischen nicht ausgeprägt $(n=334)$ und sehr stark ausgeprägt $(\mathrm{n}=927)$ ) zeigt über alle sechs ausgewählten Motive hinweg, dass die Zustimmung von Personen mit geringerer Internet-Affinität zu den genannten Gründen für den Einkauf im Ladenlokal deutlich stärker ausgeprägt ist als bei Personen mit sehr stark ausgeprägter InternetAffinität.

Ebenso wie nach den Stärken des stationären Einzelhandels wurden alle Probanden in den sechs untersuchten Kernstädten und einigen ihrer Umlandgemeinden auch nach den wichtigsten Motiven für ihren Einkauf im Internet befragt. Verglichen mit den Zustimmungswerten zu den wichtigsten Gründen für den Einkauf im Ladengeschäft erfahren die abgefragten Motive für den Einkauf im Internet (Bewertung von Antwortvorgaben) insgesamt eine weniger starke Zustimmung. Als wichtigster Grund für den Einkauf im Online-Handel wird die größere Produktvielfalt angegeben (rund $70 \%$ Zustimmung, vgl. Abbildung 4). Ungefähr jeder zweite Befragte benennt die Unabhängigkeit von Ladenöffnungszeiten, die Verfügbarkeit von Zusatzinformationen und Produktbewertungen sowie Zeit- und Wegersparnis oder auch niedrigere Preise als weitere bedeutsa- 
Abbildung 4 Bedeutende Gründe für den Einkauf im Internet

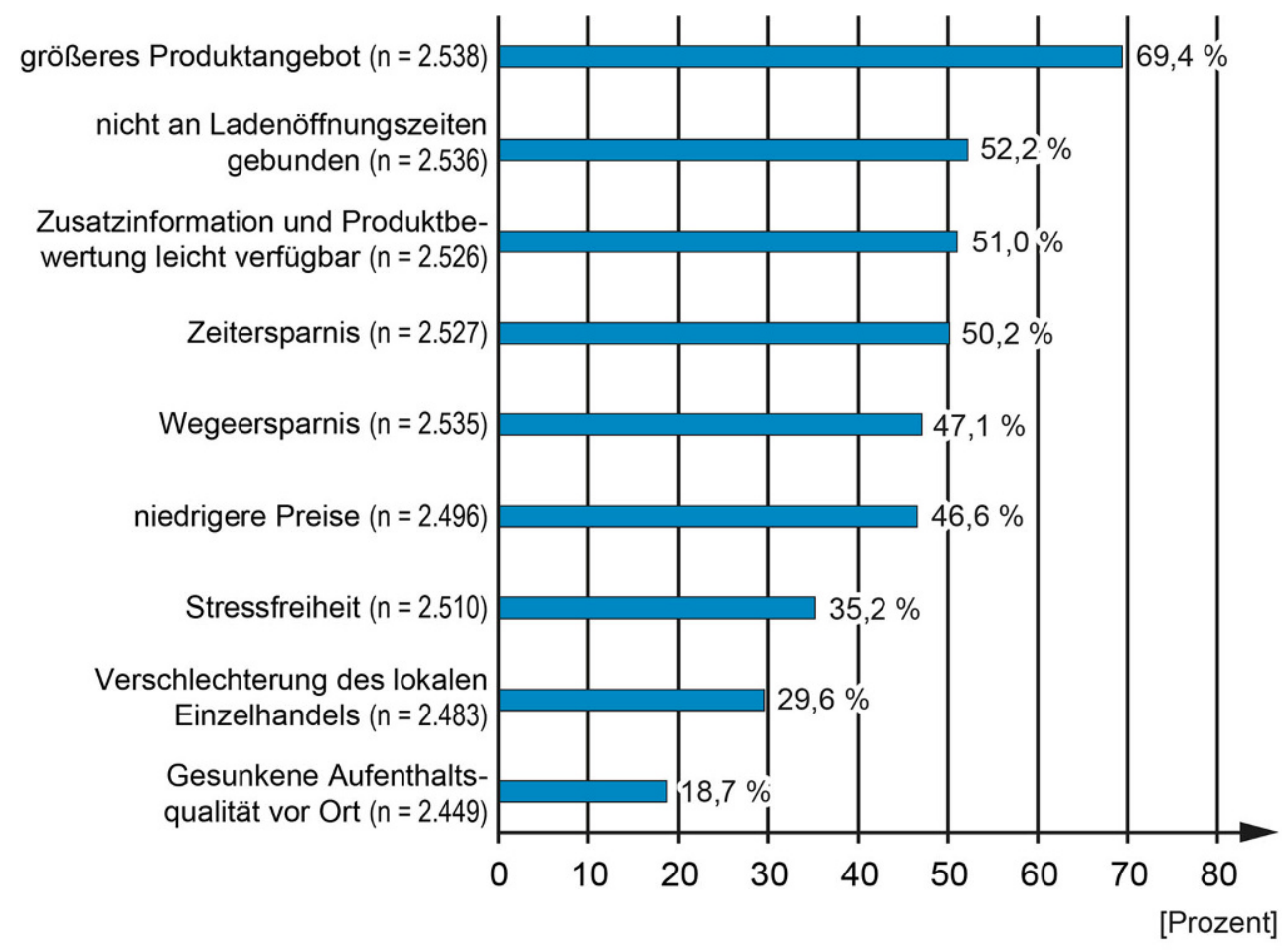

In einer Likert-Skala von 1 bis 6 wurden die beiden Antwortmöglichkeiten zusammengefasst, die die Zustimmung zu den jeweiligen Aussagen am stärksten ausdrücken (TOP 2).

Quelle: eigene Erhebungen 2016 me Motive. Weniger relevant für den Einkauf im Internet ist aus der Sicht der befragten Personen die gesunkene Aufenthaltsqualität der Innenstädte oder auch die Verschlechterung des lokalen Einzelhandelsangebotes. Dies ist ein erstes Zeichen dafür, dass räumliche Determinanten für den Einkauf im Internet eine geringere Bedeutung haben.

Differenziert man die vier bedeutendsten Gründe für den Einkauf im Internet wiederum nach den Merkmalen Entfernung, Alter und Haushaltseinkommen (vgl. Abbildung 5), zeigen sich zunächst nur geringe Unterschiede bei der Bewertung nach Entfernungstypen. Lediglich bei der Gruppe, die in innenstadtnahen Quartieren wohnt (Entfernung weniger als 10 Minuten), ist die Zustimmung zum Motiv ,größere Produktauswahl im Internet" deutlich stärker ausgeprägt. Auch ist das größere Produktangebot im Online-Handel vor allem für die Bevölkerung unter 45 Jahren besonders wichtig, wie die Altersgruppenanalyse bestätigt. Motive wie die Ungebundenheit an Ladenöffnungszeiten, die Zugänglichkeit von Zusatzinformationen und das Einsehen von Produktbewertungen sowie die Zeitersparnis beim Kauf im Internet finden aber ebenso in den Alterskohorten unter 45 Jahren deutlich höhere Zustimmungswerte als in der Generation über 45 Jahre. Eine Aufschlüsselung nach Einkommensklassen zeigt dagegen kaum Unterschiede bei der Bewertung der Gründe für den Einkauf im Internet. Tendenzi- ell ist die Zustimmung in den höheren Einkommensklassen jedoch etwas stärker ausgeprägt als beim Personenkreis mit einem monatlichen Haushaltseinkommen unter 2.000 Euro netto.

Im Hinblick auf die Rolle des Internets für das persönliche Einkaufsverhalten der insgesamt rund 2.900 befragten Personen lässt sich festhalten, dass mehr als drei Viertel aller erfassten Probanden angaben, im Internet einzukaufen und die bestellten Produkte nach Hause liefern zu lassen (vgl. Abbildung 6). Außerdem ist für fast die Hälfte der befragten Personen der Einbezug des Internets bei der Ausgestaltung des Einkaufsverhaltens maßgeblich durch die Vorabinformation im Internet über Angebote, Warenverfügbarkeit, Öffnungszeiten und Ähnliches an bestimmten Orten, in Städten oder Einkaufszentren geprägt. Dies deutet an, dass die Recherche über zu kaufende Produkte und über einen potenziellen Einkaufort zwar im Netz stattfindet, der anschließende Warenerwerb hingegen nicht zwangsläufig dort abläuft. Mehr als jeder dritte Befragte gibt zudem an, nach dem Einholen von Vorabinformationen im Internet im stationären Handel einzukaufen. Weniger charakteristisch ist es jedoch, die Vorabinformation lokal einzuholen und das Produkt im Anschluss online zu erstehen (13\% der Antworten). Dies weicht von der Annahme des stationären Handels in Betriebsbefragungen ab. Auch die Nutzung von 


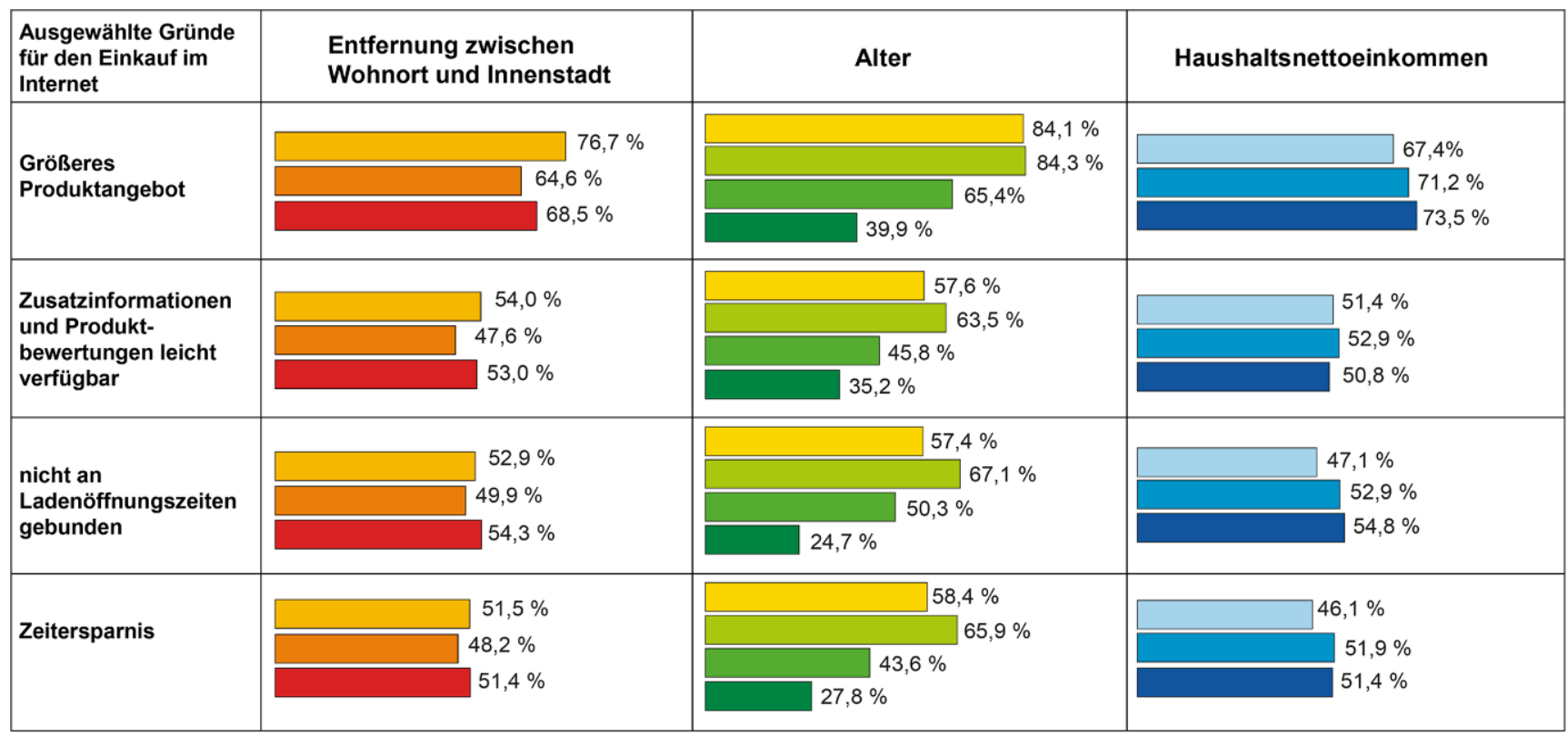

In einer Likert-Skala von 1 bis 6 wurden die beiden Antwortmöglichkeiten zusammengefasst, die die Zustimmung zu den jeweiligen Aussagen am stärksten ausdrücken (TOP 2).

\begin{tabular}{|ll}
$\square$ unter $10 \mathrm{~min}$. Entfernung $(n=776)$ & \\
\hline $\begin{array}{ll}\square \\
10 \text { bis } 30 \mathrm{~min} . \text { Entfernung }(n=1.192)\end{array}$ & 24 bis 44 Jahre $(n=793)$ \\
\hline über $30 \mathrm{~min}$. Entfernung $(n=893)$ & 45 bis 64 Jahre $(n=963)$ \\
& 65 Jahre und älter $(n=641)$
\end{tabular}

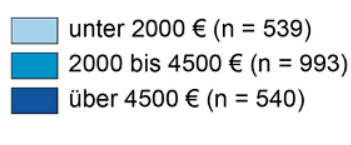

Quelle: eigene Erhebungen 2016

Abbildung 5 Ausgewählte Gründe für den Einkauf im Internet, differenziert nach Entfernungstypen, Alter und Nettoeinkommen der Haushalte

Abbildung 6 Rolle des Internets für das persönliche Einkaufsverhalten

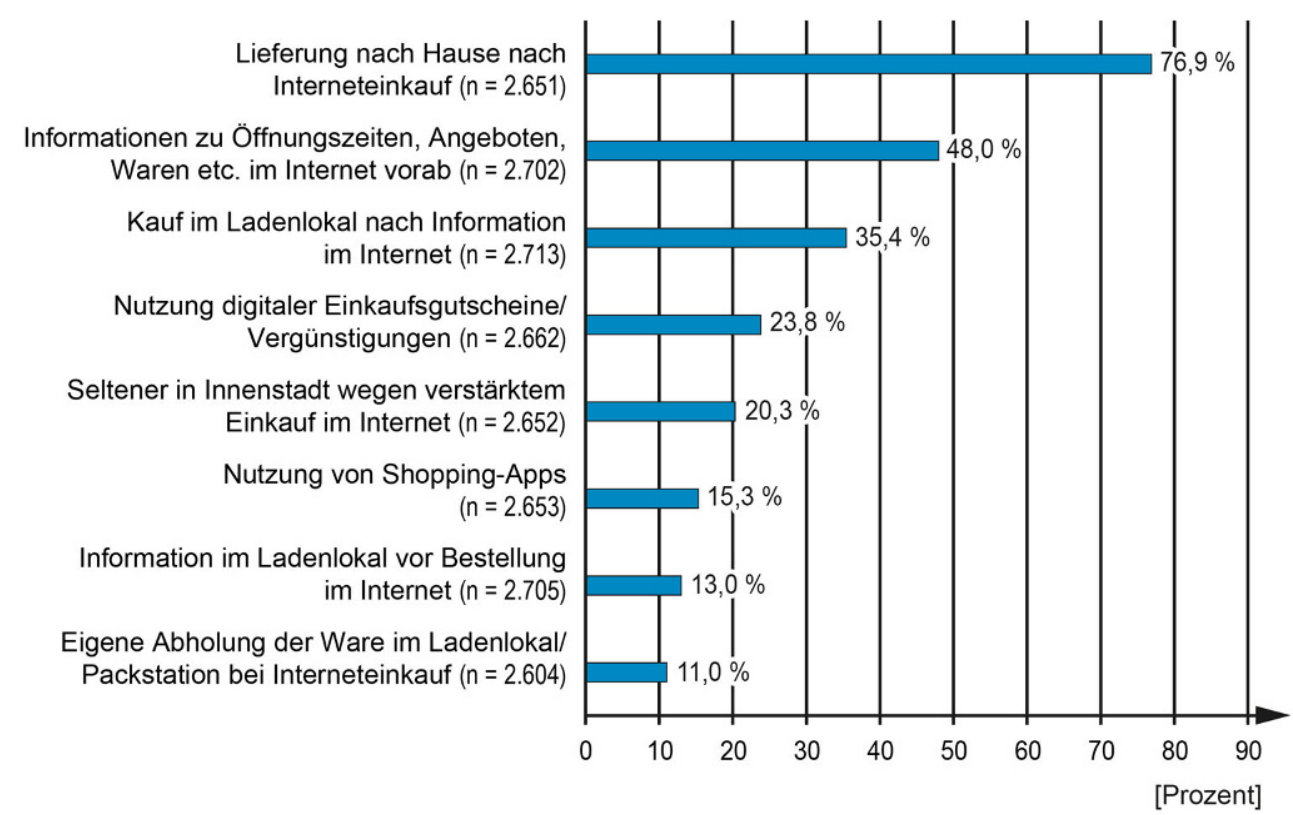

In einer Likert-Skala von 1 bis 6 wurden die beiden Antwortmöglichkeiten zusammengefasst, die die Zustimmung zu den jeweiligen Aussagen am stärksten ausdrücken (TOP 2).

Quelle: eigene Erhebungen 2016 
Shopping-Apps und der Wareneinkauf im Internet mit anschließender Abholung des Produktes im Laden bzw. einer Packstation sind bei den Befragten in den sechs untersuchten nordrhein-westfälischen Stadtregionen nicht verbreitet.

Stellt man abschließend die Stärken des stationären Einzelhandels jenen des Online-Handels gegenüber, so lässt sich Folgendes festhalten: Während die unmittelbare Möglichkeit zur Begutachtung und der sofortige Mitnahmeeffekt der Ware als bedeutendste Gründe für den Einkauf im Ladengeschäft auszumachen sind, stellt das größere Produktangebot das dominierenste Motiv für den Einkauf im Internet dar. Wie die Analyse der Rolle des Internets für das persönliche Einkaufsverhalten gezeigt hat, sind zum gegenwärtigen Zeitpunkt die Einkaufskanäle (offline/online) weiterhin als eng miteinander verkoppelte Phänomene zu erachten.

\section{Demographische und sozioökonomische Strukturen sowie Lebensstile als Determinanten des Einkaufsverhaltens}

In diesem Kapitel wird der Frage nachgegangen, welche nicht raumbezogenen Faktoren einen Einkauf im Internet wahrscheinlicher werden lassen. $\mathrm{Zu}$ diesen Faktoren zählen demographische und sozioökonomische Merkmale von Käufern sowie deren Einstellungen und Wahrnehmungen. Dabei geht es zum einen um die Relevanz eher „,klassischer“ Faktoren, wie beispielsweise Geschlecht, Alter, Erwerbstä- tigkeit, zum anderen um den Einfluss von Lebensstilen bzw. Milieus auf den Online-Einkauf. Mit diesem Abschnitt werden die zweite und dritte Forschungsfrage beantwortet (vgl. Kapitel 1).

Ausgangspunkt für die Beantwortung der Forschungsfrage zum Einfluss der demographischen und sozioökonomischen Strukturen ist die Häufigkeit des Einkaufs verschiedener Produkte im Online-Handel bzw. im stationären Einzelhandel. Wie bereits im Kapitel 5 ausgeführt, wurden für jeden Probanden die Anteile der Online-Käufe über alle Produktgruppen bzw. produktgruppenspezifisch errechnet. Diese Anteile bilden die Ausprägungen einer ersten abhängigen Variablen, mit der der Anteil von Online-Käufen zum Ausdruck gebracht wird. Aus dieser Variablen wurde eine zweite abhängige Variable abgeleitet, deren Werte sich aus einer Aggregation der Ausprägungen der ersten Variablen ableiten lassen. Die zweite Variable besitzt folgende fünf Ausprägungen bzw. Klassen: In Klasse 1 wurden diejenigen Probanden zusammengefasst, die angaben, nur im Laden einzukaufen. Der Anteil der Online-Käufe dieser Probanden liegt somit bei $0 \%$. Nimmt der Anteil einen Wert zwischen 1 und $33 \%$ ein (Klasse 2), wurden Probanden eher als Ladenkäufer denn als Online-Käufer eingestuft. Werte zwischen 34 und $66 \%$ (Klasse 3) spiegeln ein hybrides Einkaufsverhalten wider. Die Probanden kaufen also sowohl im Laden als auch im Internet ein. Schließlich weisen Werte zwischen 67 und $99 \%$ auf Käufer hin, die eher den Online-Einkauf als den Einkauf im Laden bevorzugen (Klasse 4). Ein Wert von $100 \%$ gibt an, dass ein Proband
Abbildung 7 Anteil der Online-Einkäufe an allen Einkäufen nach Produktgruppen ohne Lebensmittel

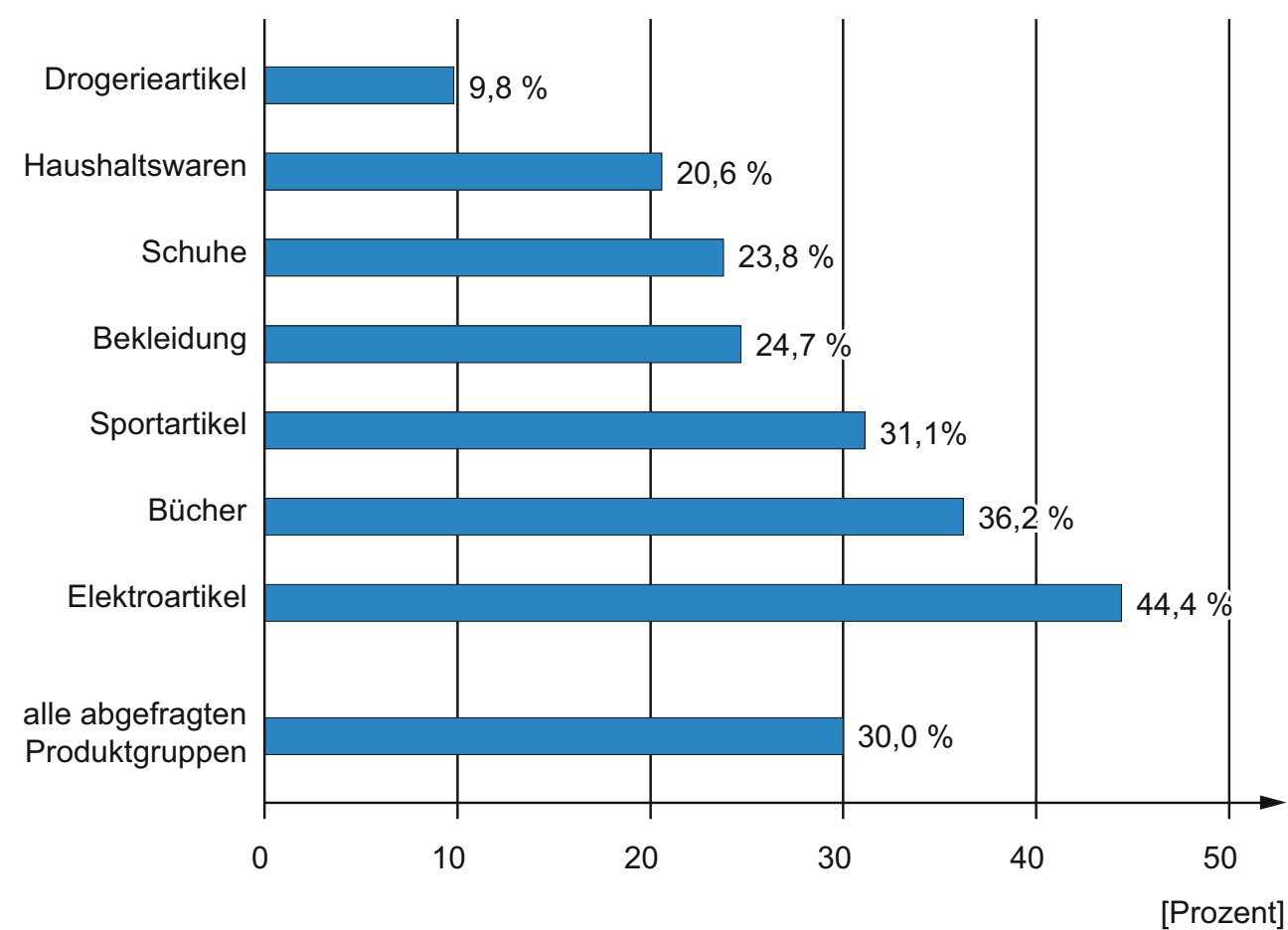

Quelle: eigene Erhebungen 2016 


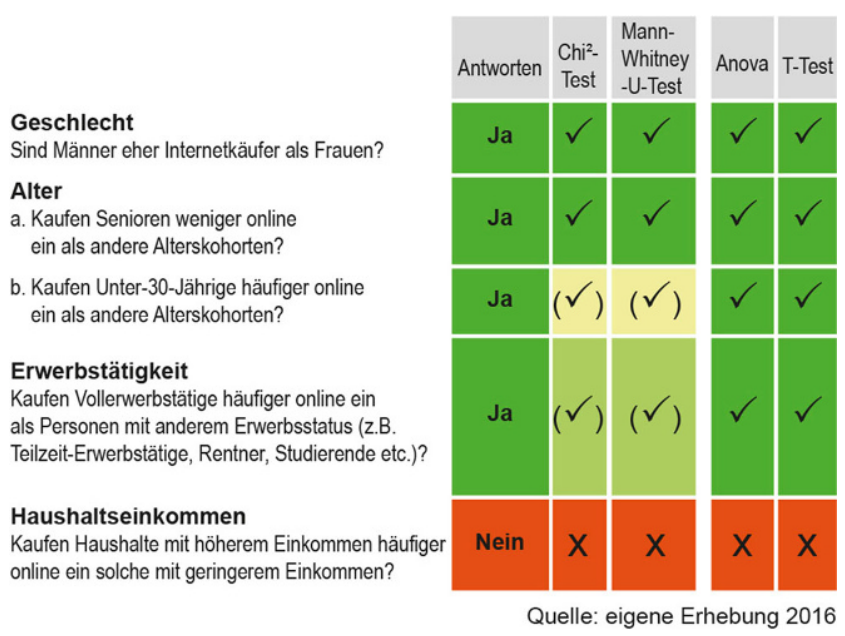

Abbildung 8 Der Einfluss demographischer und sozioökonomischer Merkmale auf den Online-Einkauf

keine Geschäfte mehr zum Einkauf aufsucht, sondern ausschließlich online seine Einkäufe tätigt (Klasse 5).

Die Differenzierung nach Online-Käufertypen zeigt, dass $30 \%$ der Kunden reine Ladenkäufer sind. Sie hatten, zumindest in den letzten drei Monaten vor dem Befragungszeitpunkt, kein Produkt online erworben. Dagegen ist der Anteil der reinen Online-Käufer mit 3,7 \% überschaubar. Allerdings variiert dieser Anteil produktspezifisch stark. Bei den Elektroartikeln etwa liegt der Anteil der reinen Online-Käufer bei fast einem Drittel. Ohne Berücksichtigung der Lebensmittel kann für die anderen sechs abgefragten Produktgruppen festgestellt werden, dass der Anteil der Online-Einkäufe bei 30,9 \% liegt (vgl. Abbildung 7). Der Ausschluss von Lebensmitteln erfolgte aus zwei Gründen: Erstens werden Lebensmittel im Vergleich zu allen anderen abgefragten Produktgruppen mehrheitlich kurzfristig nachgefragt. Eine Erhebung der Einkäufe in den letzten drei Monaten wäre daher mit großen Unsicherheiten bezüglich der Datenqualität verbunden gewesen. Zweitens befindet sich der Lebensmittel-Online-Handel noch in seinem Initialstadium. Der Umsatzanteil der Branche liegt in Deutschland noch deutlich unter einem Prozent (vgl. auch Kapitel 2 und Dederichs/Dannenberg 2017).

Die höchsten Werte in den abgefragten Produktgruppen wurden für Elektroartikel $(44,4 \%)$ verzeichnet. Bei den Büchern liegt der Anteil bei 36,2 \%. Dieser Wert berücksichtigt beide Formen der Buchlieferung, einerseits also die Lieferung des Buchs, das online bestellt und vom Paketdienst zugestellt wird, sowie andererseits das Buch, das dem Kunden in Form des digitalen Downloads verfügbar gemacht wird. Auch für Sportartikel kann ein noch leicht überdurchschnittlicher Wert festgestellt werden $(31,1 \%)$. Auf den weiteren Plätzen rangierten Bekleidung $(24,7 \%)$, Schuhe $(23,8 \%)$, Haushaltswaren $(20,6 \%)$ sowie, deutlich abgeschlagen, die Gruppe der Drogerieartikel und Arzneimittel $(9,8 \%)$.

Im Folgenden geht es um die Frage, welche demographischen und sozioökonomischen Merkmale entscheidend dafür sind, ob jemand mittels Computer oder Smartphone seine Einkäufe erledigt oder persönlich ein Geschäft aufsucht.

Die augenfälligste Differenzierung der Probanden bei den demographischen und sozioökonomischen Merkmalen ist die nach ihrem Geschlecht. Somit stellt sich im Kontext der Untersuchung die naheliegende Frage, ob es geschlechtsspezifische Unterschiede des Online-Einkaufs bzw. der Online-Käufer gibt (vgl. Abbildung 8). Methodisch wurde dabei mit vier verschiedenen Testverfahren gearbeitet: mit dem Chi-Quadrat-Test und dem MannWhitney-U-Test für nominalskalierte Variablen sowie mit der einfaktoriellen Varianzanalyse (Anova) und dem T-Test für Mittelwertunterschiede für metrisch skalierte Variablen. Die hier zugrunde gelegte Fragestellung lautet, ob Männer häufiger online einkaufen als Frauen. Die oben genannten Tests bestätigen diese Annahme eindeutig bei einer Irrtumswahrscheinlichkeit unter $0,01 \%$. Männer kaufen zwar insgesamt weniger häufig ein, aber wenn, dann eher online.

Eine zweite Variable zur Erklärung des Einkaufsverhaltens ist das Alter. Da mit zunehmendem Lebensalter die Internet-Affinität abnimmt, ist auch anzunehmen, dass einerseits Senioren in geringerem Maße online einkaufen als andere Alterskohorten und andererseits jüngere Menschen häufiger via Internet einkaufen als ältere. Auch diese beiden Annahmen können klar bestätigt werden. Allerdings muss bei zwei Testverfahren, dem Chi-Quadrat-Test und dem T-Test, eine höhere Irrtumswahrscheinlichkeit in Kauf genommen werden. Auch kann nachgewiesen werden, dass der Form der Erwerbstätigkeit ein Erklärungswert beigemessen werden kann. So kaufen Vollzeiterwerbstätige in stärkerem Umfang online ein als Teilzeiterwerbstätige oder andere Erwerbsformengruppen. Dies mag mit der Tatsache zusammenhängen, dass Vollzeiterwerbstätige kleinere Zeitbudgets für andere Grundfunktionen haben und daher den Online-Einkauf bevorzugen.

Nachweislich keinen Einfluss auf das Online-Einkaufsverhalten hat das Haushaltseinkommen. Hier scheint es allerdings in jüngerer Zeit eine Veränderung gegeben zu haben. So wurde in älteren Untersuchungen, z. B. Girard/ Korgaonkar/Silverblatt (2003), Swinyard/Smith (2003), Fa$\mathrm{rag} /$ Weltevreden/van Rietbergen et al. 2006), stets die Bedeutung des Haushaltseinkommens als relevanter Einflussfaktor betont. Mögliche Gründe hierfür sind sinkende Kosten für die Nutzung des Internets sowie die rasante Verbreitung frei zugänglicher WLAN-Netze und mobiler Endgeräte, insbesondere Tablets und Smartphones. Beispielsweise waren im Jahre 2011 erst $27 \%$ der Bundesbürger über 14 Jahre im Besitz eines Smartphones. Für das Jahr 2017 hin- 
gegen wurde ein Anteil von $81 \%$ angenommen (EHI Retail Institute 2017: 27).

Jeder Käufer lässt sich einer Lebensstilgruppe bzw. einem Milieu zuordnen. Je nach Quelle und Methode lässt sich eine mehr oder weniger große Zahl unterschiedlicher Lebensstilgruppen identifizieren. In der Untersuchung haben wir die Lebensführungstypologie nach Otte (2005) angewandt. Dabei sind das „Ausstattungsniveau“ - gemeint hat Otte hiermit die Ausstattung mit finanziellen Ressourcen und mit Bildungsabschlüssen - und die „Modernität bzw. die biographischen Perspektiven" die beiden leitenden Dimensionen, um die Lebensführung der Befragten in neun sogenannten Lebensführungstypen zusammenzuführen. Diese neun Typen ergeben sich aus der Kombination von niedrigem, mittlerem und gehobenem Ausstattungsniveau sowie traditioneller, teilmoderner und moderner biographischer Perspektive. Dazu wurden zehn Fragen aus dem Konzept von Otte (2005) übernommen.

Jeder Proband wurde nun genau einer der neun von Otte ausgewiesenen Lebensführungstypen zugeordnet. Die $\mathrm{Zu}$ ordnung der Probanden zu den Lebensführungstypen erfolgte in Anlehnung an das von Otte (2005) vorgeschlagene und validierte Verfahren. Dabei wurden den Befragten sowohl zur Erfassung des Ausstattungsniveaus als auch des Modernitätsniveaus jeweils fünf Aussagen vorgelegt. Die Probanden sollten diese Statements mit „trifft voll und ganz zu“, „trifft eher zu“, ,trifft nicht zu“ und ,trifft überhaupt nicht zu" bewerten. Für jede Antwortkategorie wurden ein, zwei, drei oder vier Punkte vergeben. Die Punkte wurden schließlich für jede Dimension summiert und gemittelt. Somit ergaben sich zwei Mittelwerte, einer für das Ausstattungs- und einer für das Modernitätsniveau. Diese Mittelwerte bildeten die Grundlage für die Zuordnung von Probanden in eine der neun Lebensführungsgruppen (für weitere Details vgl. Otte 2005).

Für die neun Kohorten wurde nun mit den oben genannten Testverfahren auf Abweichungen des Online-Einkaufens geprüft und diese auf Signifikanz getestet. Folgende Ergebnisse lassen sich aufgrund dieser Vorgehensweise feststellen (vgl. Abbildung 9): Die Hedonisten und Unterhaltungssuchenden sind die beiden Lebensstilgruppen, die signifikant häufiger als andere Gruppen online einkaufen. Dabei sind für beide Gruppen Gründe entscheidend, die zum einen mit Kosten, zum anderen mit Bequemlichkeit verbunden sind. So geben $64,1 \%$ der Hedonisten und Unterhaltungssuchenden an, dass für sie die niedrigeren Preise beim Internet-Einkauf wichtig bzw. sehr wichtig sind. Für die anderen Gruppen wird dagegen hierfür ein Anteil von nur 49,2\% ermittelt. Auch der stressfreie Einkauf im Netz sowie die Zeit- und Wegersparnis sind für die Hedonisten und Unterhaltungssuchenden wichtiger als für die anderen Gruppen. So verweisen beispielsweise 53,9\% aus den beiden Gruppen auf den stressfreien und somit beque-

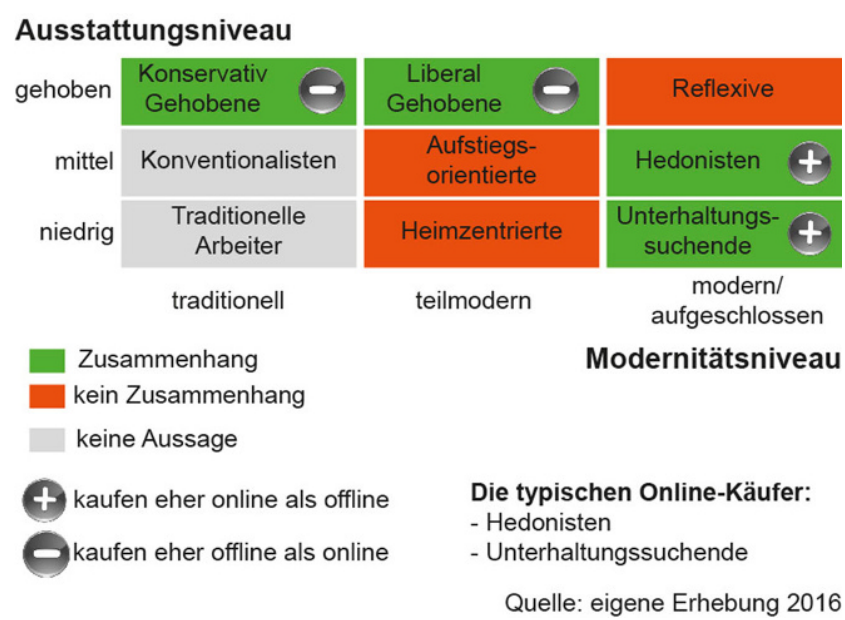

Abbildung 9 Der Einfluss von Lebensstilen auf den Online-Einkauf

men Online-Einkauf, der für sie wichtig bzw. sehr wichtig sei. In den anderen Lebensstilgruppen liegt dieser Anteil im Durchschnitt bei nur 42,8\%. Alle hier genannten Unterschiede konnten mittels Chi-Quadrat-Tests als signifikant eingestuft werden.

Die konservativ und liberal gehobenen Milieus zählen dagegen zu den stärker auf den Ladeneinkauf ausgerichteten Gruppen. Für die restlichen fünf Milieus können keine eindeutigen Zusammenhänge zwischen Einkaufsverhalten und Milieuzugehörigkeit festgestellt werden.

Es bleibt festzuhalten, dass zum einen der Online-Einkauf mittlerweile alle gesellschaftlichen Gruppen erfasst hat. Preiswerte, standardisierte Massenprodukte werden vor allem von aufgeschlossenen und experimentierfreudigen Personen mittlerer und unterer sozialer Schichten nachgefragt, während Personen mit hoher Bildung und Kaufkraft, die eher als konservativ bzw. zurückhaltend einzuordnen sind, stärker den Ladeneinkauf favorisieren. Sie mit einem anspruchsvollen Ambiente und einer kompetenten Beratung an das Geschäft zu binden, sollte ein Ziel des stationären Handels sein. Damit verbunden könnte in letzter Konsequenz auch eine qualitative Aufwertung der Innenstädte sein.

\section{Räumliche Faktoren in den sechs Stadtregionen als Determinanten des Einkaufsverhaltens}

Die beiden letzten Forschungsfragen dieses Beitrages (vgl. Kapitel 1) zielen darauf ab, den Einfluss der räumlichen Faktoren auf das Einkaufsverhalten der Konsumenten zu untersuchen. Dazu wird zunächst die Attraktivität der Innenstädte der sechs untersuchten nordrhein-westfälischen Stadtregionen als Einzelhandelsstandorte betrachtet, die sich durch drei Indikatoren bestimmen lässt (vgl. Abbil- 
Abbildung 10 Bewertung der Innenstädte hinsichtlich der Indikatoren Einzelhandelsangebot, Attraktivität und Erreichbarkeit

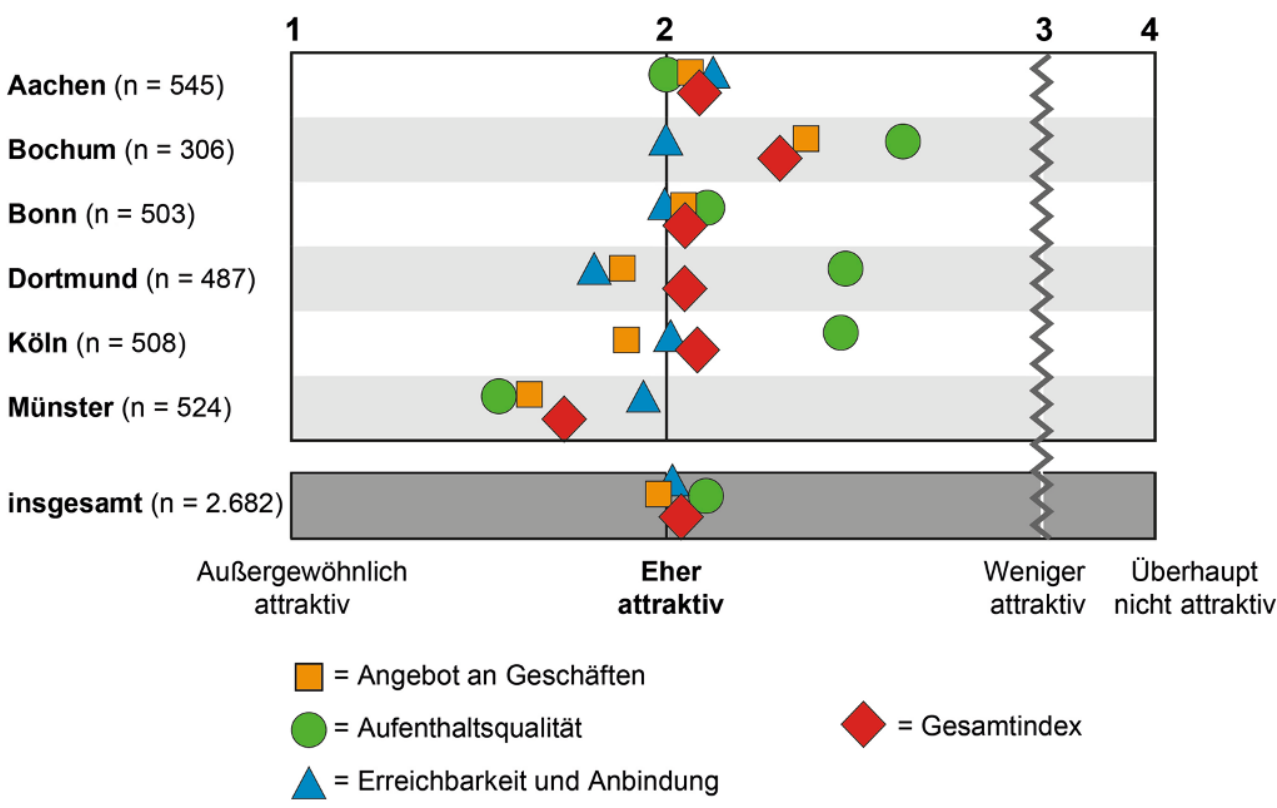

Quelle: eigene Erhebungen 2016 dung 10). Hinsichtlich des Angebotes an Einzelhandelsgeschäften werden die sechs Innenstädte der Oberzentren als „eher attraktiv“ eingeschätzt, wobei die Bewertung (angegeben als Mittelwerte einer Benotung von ,außergewöhnlich attraktiv“ (1) bis ,überhaupt nicht attraktiv“ (4)) von 1,7 (Münster) bis 2,4 (Bochum) reicht. Bei der Bewertung von Aufenthalts- und Verweilqualität vor Ort (z. B. bezogen auf Ausstattung und Architektur) rangiert die Innenstadt des solitären Oberzentrums Münster $(1,6)$ mit deutlichem Abstand vor Aachen (2,0) und Bonn (2,2). Die City der Ruhrgebietsstadt Bochum belegt bei diesem Bewertungskriterium den letzten Platz $(2,7)$ und schneidet lediglich beim Indikator Erreichbarkeit (abgefragt waren hier insbesondere ÖPNV-Anbindung und Parkplatzmöglichkeiten) etwas besser $a b$, als das bei diesem Indikator letztplatzierte Aachen (2,2). Aus der Sicht der Befragten weist die Dortmunder Innenstadt die beste Erreichbarkeit auf $(1,9)$. Generiert man durch Summieren und erneuter Durchschnittsbildung aus den drei genannten Indikatoren einen Gesamtindikator, so ergibt sich - bezogen auf die Attraktivität der untersuchten Einkaufsstädte - ein deutlicher Vorsprung von Münster $(1,7)$ vor Dortmund, Aachen, Bonn und Köln, die nahezu gleichauf liegen und insgesamt als eher attraktiv bewertet werden $(2,1)$ sowie der letztplatzierten Innenstadt Bochums $(2,4)$.

Im Weiteren geht es um die Frage, ob die Attraktivität der Innenstädte der Stadtregionen, in denen die Befragten jeweils wohnen, Einfluss auf die Häufigkeit des Einkaufs im stationären Einzelhandel bzw. im Online-Handel ausübt (vgl. Abbildung 11). Dazu kann man zunächst die Unterschiede zwischen den sechs Stadtregionen betrachten, die in die Untersuchung einbezogen wurden. Dahinter steht die
Überlegung, dass eine attraktive Innenstadt mit einem guten Angebot im Einzelhandel dazu führt, dass die Bewohner genau diese stationären Angebote dem Online-Handel vorziehen. Im Wettbewerb der Städte könnten also attraktivere Innenstädte einen Faktor darstellen, dem Online-Handel zu begegnen. Um diese These zu untermauern, wird noch einmal die abgeleitete Variable zum Verhältnis von Ladenkäufern zu Online-Käufern genutzt (vgl. Kapitel 4 und 6). Zur Vereinfachung werden hier nur drei Klassen betrachtet. In der ersten Klasse werden alle Probanden zusammengefasst, die nur im Laden einkaufen bzw. bei denen der Wert zwischen einem Prozent und $33 \%$ liegt. Die insgesamt 1.372 Probanden werden als ,,eher Offline-Käufer“ bezeichnet. Die zweite Klasse mit 675 Probanden spiegelt hier mit Werten zwischen 34 und $66 \%$ das hybride Einkaufsverhalten wider. In der dritten Klasse befinden sich schließlich die 325 Probanden der Befragung, die überwiegend oder sogar ausschließlich online einkaufen.

Im Ergebnis zeigen sich zwischen den sechs Stadtregionen keine großen Unterschiede beim Verhältnis von Onlinezu stationärem Einkauf (vgl. Abbildung 11). Noch kaufen in allen Stadtregionen mehr als die Hälfte der Kunden häufiger offline ein. Bei den stärker online orientierten Käufern der dritten Klasse haben die Stadtregionen Bochum und Köln nur leicht höhere Anteile als die Stadtregionen Aachen, Bonn, Dortmund und Münster. Die nur geringen Unterschiede legen die Schlussfolgerung nahe, dass die unterschiedlichen Einschätzungen zur Attraktivität der jeweiligen Kernstädte (vgl. Kapitel 5) sich kaum auf das OnlineEinkaufsverhalten auswirken. Die deutlich unterschiedlichen Bewertungen der Angebote an Geschäften, der Aufenthaltsqualität sowie der Erreichbarkeit und Anbindung in 


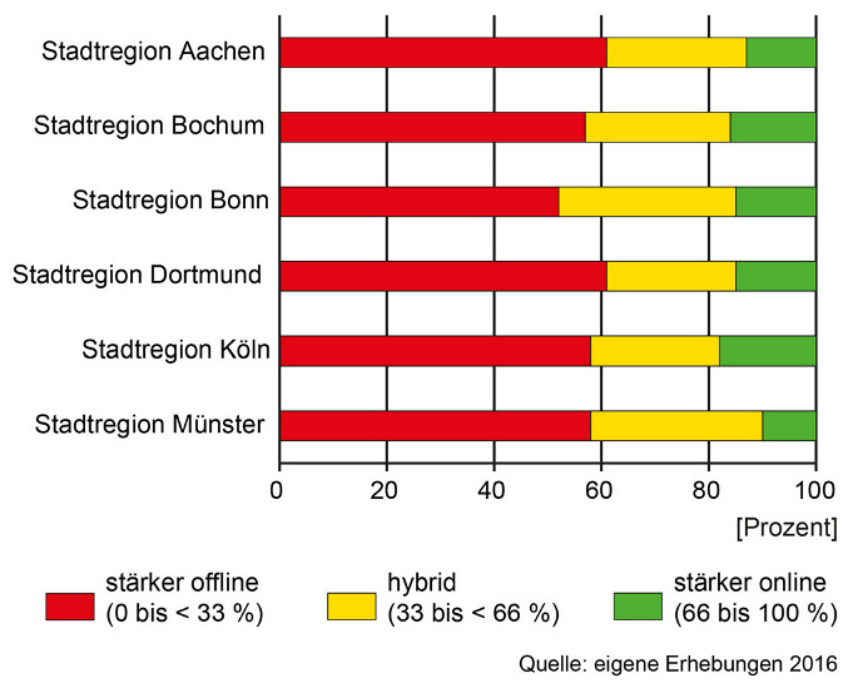

Abbildung 11 Das Verhältnis von Online- und Offline-Einkauf nach den sechs Stadtregionen

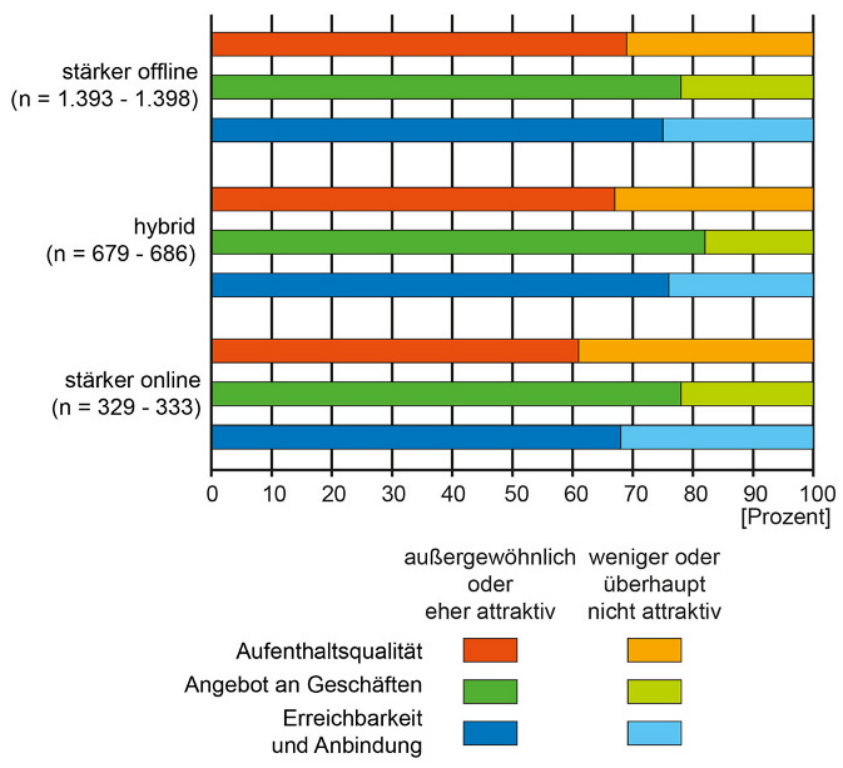

Quelle: eigene Erhebungen 2016

Abbildung 12 Der Zusammenhang von Online- und Offline-Einkauf bei der Bewertung der Attraktivität der Innenstädte

den sechs Stadtregionen (vgl. Kapitel 5) ist hier am Verhältnis von Online- zu Offline-Käufern nicht ablesbar.

Betrachtet man das Verhältnis der Online- zu den Offline-Käufern bei der Bewertung der Attraktivität der Innenstädte unabhängig von den Stadtregionen für alle Befragten in den drei beschriebenen Intensitätsklassen, zeigen sich ebenfalls nur leichte Unterschiede hinsichtlich der drei Indikatoren, die die Attraktivität bestimmen (vgl. Abbildung 12). Die Befragten, die „stärker online“ einkaufen, empfinden die Aufenthalts- und Verweilqualitäten der Innenstädte zu fast $40 \%$ als weniger oder überhaupt nicht attraktiv, während die „stärker offline“ orientierten Befrag-

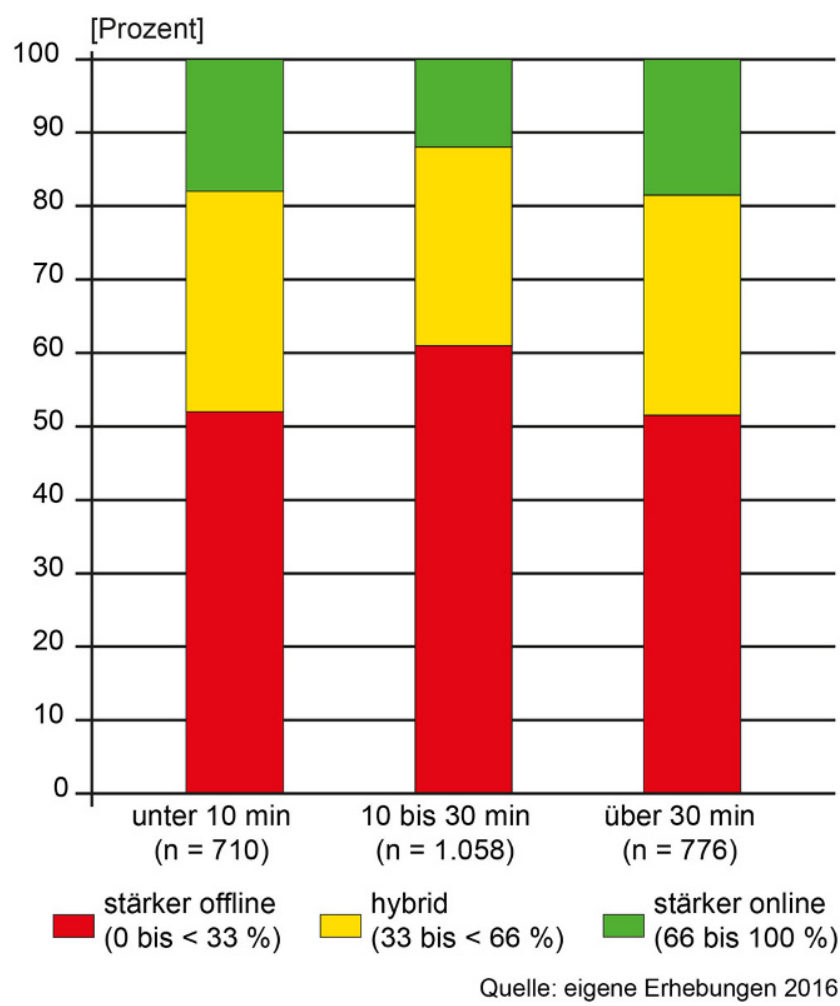

Abbildung 13 Zusammenhang von Online- und Offline-Einkauf und Fahrzeit mit dem öffentlichen Verkehr in die Innenstadt

ten hier auf einen Wert von nur knapp über $30 \%$ kommen. Ein Chi-Quadrat-Test zeigt, dass diese Unterschiede zwar signifikant sind, die Stärke des Zusammenhangs allerdings eher schwach ausgeprägt ist. Eine vergleichbare Einschätzung lässt sich auch für die Erreichbarkeit und Anbindung der Innenstädte feststellen. Hier sind es rund $32 \%$ der stärker online orientierten Käufer, die die Erreichbarkeit und Anbindung für wenig oder überhaupt nicht attraktiv halten, im Gegensatz zu den rund $24 \%$ der offline orientierten Käufern. Der statistische Test ergibt auch hier eine Signifikanz. So gibt es leichte Zusammenhänge zwischen der Attraktivität der Innenstädte und dem bevorzugten Einkaufsverhalten im stationären Einzelhandel bzw. im Online-Handel.

Neben den Zusammenhängen zwischen der Attraktivität der Innenstädte geht es bei den räumlichen Determinanten des Einkaufsverhaltens im Weiteren um die Entfernung zwischen dem Wohnort der Befragten und der Entscheidung, offline bzw. online einzukaufen. Es könnte die These vertreten werden, dass mit einer größeren Entfernung zur Innenstadt (gemessen in den Fahrminuten mit dem öffentlichen Verkehr) die Angebote dort weniger genutzt werden und stattdessen der Online-Einkauf attraktiver wird. Bei diesem zweiten räumlichen Aspekt zeigen sich aber fast keine Unterschiede zwischen den Entfernungsklassen (vgl. Abbildung 13). Unter den 776 Befragten, die in mehr als 30 Minuten Entfernung zu den Innenstädten wohnen, sind 
mit etwa $16 \%$ fast genauso viele stärker online orientierte Käufer wie bei den 710 Befragten, die in unter zehn Minuten Entfernung zur Innenstadt wohnen. Dort sind es $15 \%$ der Befragten. Zusammenhänge zwischen der Lage des Wohnorts zur Innenstadt und dem Online- bzw. Offline-Einkaufsverhalten sind somit nicht feststellbar. Ein Chi-Quadrat-Test bestätigt dies.

\section{Fazit: Folgerungen für planerische Handlungsansätze}

Zunächst soll noch einmal auf die Besonderheiten der durchgeführten Untersuchung hingewiesen werden. Dies gilt insbesondere für den Umstand, dass sich alle betrachteten Untersuchungsgebiete in stärker verdichteten Stadtregionen des Landes Nordrhein-Westfalen befinden. Möglicherweise gewinnen räumliche Determinanten an Erklärungskraft, wenn auch (eher) ländliche Regionen in größerer Entfernung zu einem (attraktiven) Oberzentrum untersucht werden. Diesbezüglich besteht weiterer Forschungsbedarf. Weiterhin ist es wünschenswert, sich unter Berücksichtigung der Erkenntnisse aus früheren Arbeiten näher mit dem Begriff der „Attraktivität von Innenstädten“ auseinanderzusetzen (z. B. Teller/Reutterer 2008; Teller/ Elms 2010). Für wirkungsvolle Maßnahmen ist es notwendig, möglichst detailliert zu wissen, was sich genau hinter diesem Schlagwort verbirgt. Angesichts der Komplexität des Begriffs stoßen Befragungen hierbei an Grenzen, sodass - zumindest als wichtige Ergänzung - andere methodische Untersuchungsansätze wie qualitative Interviews und teilnehmende Beobachtungen zur Anwendung kommen müssen.

Die in diesem Beitrag vorgestellten empirischen Ergebnisse verdeutlichen zum einen die zunehmende Verbreitung des Online-Handels als Einkaufskanal, belegen aber auch, dass die ganz überwiegende Zahl der Einkäufe immer noch stationär (offline) erfolgt.

Unter den betrachteten demographischen und sozioökonomischen Merkmalen zeigen sich vor allem beim Alter auffällige Unterschiede, was das Einkaufsverhalten und die hierfür vorgebrachten Gründe betrifft. Neben den jungen Erwachsenen sind es insbesondere die Menschen in der „Rushhour“ des Lebens, die offensichtlich die Vorzüge des Internet-Einkaufs schätzen. Die in der Untersuchung aufgezeigten Unterschiede zwischen den Altersgruppen beim Einkaufsverhalten, die im Wesentlichen auf Kohorteneffekte zurückzuführen sind, sprechen dafür, dass der OnlineEinkauf voraussichtlich auch in den kommenden Jahren weiter an Bedeutung gewinnen wird. Bemerkenswert im Hinblick auf die weitreichende Akzeptanz des Online-Handels ist außerdem, dass entgegen früherer Studien keine
Unterschiede nach dem Haushaltseinkommen festgestellt wurden (vgl. Kapitel 5 und 6).

Ein besonderes Augenmerk der Untersuchung lag auf der Frage nach möglichen räumlichen Determinanten des Online-Einkaufs. Tatsächlich ergeben sich beim Einkaufsverhalten keine signifikanten Unterschiede in einem Vergleich zwischen den Großstadtregionen bzw. den näher betrachteten Quartieren. Zumindest die Entfernung zur Innenstadt hat innerhalb der fünf untersuchten Stadtregionen und Entfernungsklassen keinen Einfluss auf die Wahl zwischen Online- und Offline-Einkauf (vgl. Kapitel 6). Es sind - wie die Untersuchung belegt - vielmehr die Lebensstile und sozioökonomische Determinanten, die das Einkaufsverhalten beeinflussen.

Im Hinblick auf die Auswertungsergebnisse scheint damit eine Botschaft klar: Auch in Stadtregionen mit einer besonders attraktiven Innenstadt und in innenstadtnahen Untersuchungsräumen kommt dem Online-Einkauf eine große Bedeutung zu, da er den Menschen - zumindest in gewissem Maße unabhängig von ihrem Wohnstandort - bestimmte Vorteile bietet. Hierzu zählen nach den Befragungsergebnissen vor allem die Zeitersparnis und Unabhängigkeit beim Einkauf sowie das größere Produktangebot und verfügbare Produktinformationen. Die mit der Untersuchung gewonnenen Erkenntnisse unterstützen damit insgesamt die vorliegenden Resultate aus internationalen Studien, die eher für eine vergleichsweise geringe Relevanz von räumlichen Determinanten sprechen (vgl. Kapitel 3). Gleichwohl zeigen sich plausible - wenn auch nicht besonders große Unterschiede bei den Bewertungen zur Attraktivität der Innenstädte, die offline-orientierte Käufer positiver einstufen als online-orientierte (vgl. Kapitel 7).

Es stellt sich nun die wichtige Frage, welche Schlussfolgerungen die Akteure der Stadtentwicklung aus diesen Untersuchungsergebnissen ziehen sollten und welche Handlungsansätze sich ihnen bieten. Die Kommunen haben den gesetzlichen Auftrag der Daseinsvorsorge. Die Stärkung und Sicherung der Innenstädte einschließlich des stationären Einzelhandels mit seiner Versorgungsfunktion ist eine zentrale Aufgabe der Stadtentwicklungspolitik und damit auch der Stadtplanung. Dies unterstreicht das Handlungserfordernis und dessen Legitimation. Bei der Diskussion um kommunale Handlungsansätze sind einige wichtige Aspekte zu beachten: Zwar stellt das Einkaufen mithilfe des Internets für weite Teile der Bevölkerung eine gebräuchliche Praxis dar, die meisten Einkäufe werden aber nach wie vor im stationären Handel getätigt und nicht alle Bevölkerungsgruppen sind internetaffin. Gleichwohl erhöht die Umsatzverlagerung in den Online-Handel den Standortwettbewerb der Kommunen untereinander: Wenn weniger Kaufkraft in den stationären Handel fließt, wird der Wettbewerb um die verbleibende Kaufkraft entsprechend stärker. Das Einkaufsverhalten ist sehr dynamisch, die weitere Entwicklung aber 
nicht hinreichend vorhersehbar, sodass die Zentrenstärkung auch vor diesem Hintergrund von Bedeutung ist. Dabei sind Innenstädte und Stadtteilzentren viel mehr als der dort angebotene Einzelhandel. Sie werden entscheidend durch ihr städtebauliches Erscheinungsbild und ihre Funktionsvielfalt geprägt.

Diese Erkenntnisse begründen eine neue Akzentsetzung bei den städtischen Handlungsansätzen. Geeignet erscheint vor allem eine Doppelstrategie: zum einen die Sicherung und Stärkung attraktiver Innenstadtzentren und zum anderen eine standortangepasste Strategie zur Reaktion auf das veränderte Einkaufsverhalten und zur Nutzung der digitalen Möglichkeiten.

Auch wenn die präsentierten Befunde zunächst Zweifel daran aufkommen lassen könnten, dass Maßnahmen zur Steigerung der Attraktivität von Innenstädten tatsächlich dazu geeignet seien, der weiteren Verbreitung des OnlineEinkaufs und dem damit verbundenen Bedeutungsverlust des stationären Einzelhandels entgegenzuwirken, sollten die Kommunen angesichts des sich verschärfenden Standortwettbewerbs in ihren Anstrengungen zur Innenstadtattraktivierung nicht nachlassen. Attraktive Innenstädte - so die weit verbreitete Vermutung (vgl. DST 2016; BBSR 2017) - dürften günstigere Voraussetzungen aufweisen, sich im durch den Online-Handel beschleunigten Strukturwandel zu behaupten. Nimmt man das Leitbild der europäischen Stadt im Sinne der ,Leipzig Charta zur nachhaltigen europäischen Stadt“ (2007) ernst, prägen die (historischen) Stadtzentren das kulturelle Erbe mit ihrer urbanen Nutzungsund Gestaltqualität auch zukünftig.

Die Befragungsergebnisse zeigen darüber hinaus auch, dass die Unterstützung des lokalen Handels ein weit verbreitetes Motiv für den Einkauf im Ladengeschäft ist. Ebenfalls von Bedeutung - wenn auch nachgelagert - sind die Beratungsqualität vor Ort und das Einkaufserlebnis in der Innenstadt. Darüber hinaus gaben mehr Befragte an, sich zunächst im Internet zu informieren und dann im Ladengeschäft zu kaufen, als dies andersherum der Fall ist (vgl. Kapitel 5). Um diese hohe Wertschätzung bei den Kunden aufrechtzuerhalten und regelmäßig neue Ansätze für einen Innenstadtbesuch zu schaffen, sind Emotionen, Atmosphäre und zwischenmenschliche Kommunikation in den Innenstädten und in den Geschäften, Freundlichkeit und Service, ein zeitgemäßes und zielgruppengerechtes Sortiment, eine einladende, moderne Verkaufsflächen- und Schaufenstergestaltung, die Gestaltung des Einkaufserlebnisses mit Aktionen und Veranstaltungen sowie eine gezielte Kundenansprache unter Einbeziehung der neuen Medien wichtige Ansätze. Aus kommunaler Sicht ist es deshalb wichtig, gemeinsam mit allen in den Innenstädten handelnden Akteuren darauf hinzuwirken, dem Innenstadteinkauf einen Mehrwert gegenüber dem (reinen) Internet-Einkauf zu bieten. (vgl. Beckmann/Hangebruch 2016).
Gleichzeitig dürfte es in der gegenwärtigen Situation entscheidend darauf ankommen, inwieweit dem Einzelhandel in den Innenstädten eine konsequente Integration der verschiedenen Vertriebskanäle unter Einbeziehung der Ladengeschäfte vor Ort gelingt. Heinemann (2017) spricht in diesem Zusammenhang auch von einer „Neuerfindung des stationären Einzelhandels“, was die Reichweite der damit verbundenen Veränderungen verdeutlicht. Folgerichtig sollte ein Schwerpunkt der aktuellen Stadtentwicklungspolitik darin bestehen, die lokalen Händler in ihren Bemühungen zu unterstützen, sich erfolgreich in ,beiden Welten' zu bewegen und ein kanalübergreifendes Einkaufserlebnis zu bieten. Die mit der durchgeführten Untersuchung aufgezeigten Einstellungen der befragten Personen (vgl. Kapitel 5) unterstreichen die Bedeutung dieses Weges und lassen den Wunsch erkennen, die unterschiedlichen Stärken von Offline und Online beim Einkaufen miteinander zu kombinieren.

In einigen Innenstädten und vor allem in Nebenzentren ist trotz aller Bemühungen von einem Rückgang der Verkaufsflächen auszugehen. Nicht nur hier ist die Stärkung der Multifunktionalität der Innenstädte von besonderer Bedeutung. Funktionsvielfalt und Nutzungskopplung - in der Haushaltsbefragung wurde die Kopplung des Einkaufs mit anderen Innenstadtunternehmungen als wichtiges Argument für den Einkauf im Ladengeschäft genannt (vgl. Kapitel 5) - bieten Ansatzpunkte für das kommunale Handeln. Nicht immer werden Ladengeschäfte wieder durch Einzelhandel besetzt werden können. Auch an diesen Standorten sind Maßnahmen zur Steigerung der Attraktivität für die Akteure der Stadtentwicklung ein wichtiges Thema. Allerdings ist hier ein deutliches Umdenken gefordert, da auf der Grundlage einer realistischen Bestandsaufnahme verstärkt andere Nutzungen in neue konzeptionelle Überlegungen einbezogen werden müssen. Dazu gehören Angebote aus den Bereichen Gastronomie, Kultur und Sport sowie Bildung und Gesundheit (vgl. Beckmann/Hangebruch 2016). Die Erarbeitung integrierter Handlungs- bzw. Stadtentwicklungskonzepte kann hierbei wertvolle Unterstützung leisten. Aus städtischer Sicht ist es entsprechend wichtig, auch den Flächen- und Nutzungswandel im Kontext der Digitalisierung aller Lebensbereiche zu begleiten und proaktiv zu steuern.

Wir können den Wind nicht ändern, aber die Segel anders setzen. (Aristoteles)

Danksagung Wir danken den Studierenden an den Geographischen Instituten in Aachen, Bochum, Bonn, Münster und Köln sowie an der Fakultät Raumplanung der Technischen Universität Dortmund für ihren Einsatz. Im Laufe des Wintersemesters 2016/2017 haben sie im Rahmen ihrer Studienprojekte die Befragungen durchgeführt und inhaltliche Anregungen für diesen Beitrag gegeben. Bei der Auswertung 
und redaktionellen Bearbeitung waren uns Miriam Gruber und Julia Müller eine große Unterstützung.

\section{Literatur}

BBSR - Bundesinstitut für Bau-, Stadt- und Raumforschung (Hrsg.) (2017): Online-Handel - Mögliche räumliche Auswirkungen auf Innenstädte, Stadtteil- und Ortszentren. Bonn. = BBSR-OnlinePublikation 08/2017.

Beckmann, R. M.; Hangebruch, N. (2016): Lokale Online-Marktplätze: ein Ansatz zur Vernetzung von Fußgängerzone und Innenstadt. In: Forum Wohnen und Stadtentwicklung 8, 1, 7-12.

BMWi - Bundesministerium für Wirtschaft und Energie (2017): Neue Perspektiven für den Einzelhandel. Handlungsempfehlungen der Dialogplattform Einzelhandel. Berlin.

Brashear, T. G.; Kashyap, V.; Musante, M. D.; Donthu, N. (2008): A six-country comparison of the internet shopper profile. In: Revista de Ciências da Administração 10, 21. 54-75. doi: 10.5007/21758069.2008v10n21p54

Burroughs, R. E.; Sabherwal, R. (2002): Determinants of Retail Electronic Purchasing: A Multi-Period Investigation. In: Infor - Information Systems and Operational Research 40, 1 35-56. doi: $10.1080 / 03155986.2002 .11732640$

Cao, X.; Douma, F.; Cleaveland, F. (2010): Influence of E-Shopping on Shopping Travel. In: Transportation Research Record 2157, 147-154. doi: 10.3141/2157-18

Dederichs, S.; Dannenberg, P. (2017): Vom Nischenmarkt in die Expansion: Die aktuelle Dynamik des deutschen Online-Lebensmitteleinzelhandels im Kontext der Transitionsforschung. In: Dannenberg, P.; Willkomm, M.; Zehner, K. (Hrsg.): Einzelhandel in Deutschland - aktuelle Dynamiken im Kontext neuer Rahmenbedingungen. Mannheim, 59-82. = Geographische Handelsforschung 25.

Dijst, M.; Farag, S.; Schwanen, T. (2008): A Comparative Study of Attitude Theory and other Theoretical Models for Understanding Travel Behaviour. In: Environment and Planning A 40, 4, 831847. doi: $10.1068 / \mathrm{a} 39151$

DST - Deutscher Städtetag (2016): Zukunft von Stadt und Handel. Diskussionspapier des Deutschen Städtetages. Berlin/Köln. http:// www.staedtetag.de/imperia/md/content/dst/diskussionspapier_ zukunft_stadt_handel_072016.pdf (12.01.2018).

EHI Retail Institute (2017): EHI Handelsdaten aktuell 2017. Köln.

Farag, S.; Dijst, M.; Lanzendorf, M. (2003): Exploring the Use of E-Shopping and its Impact on Personal Travel Behavior in the Netherlands. In: Transportation Research Record 1858, 47-58. doi: 10.3141/1858-07

Farag, S.; Krizek, K. J.; Dijst, M. (2006): E-Shopping and its Relationship with In-store Shopping: Empirical Evidence from the Netherlands and the USA. In: Transport Reviews 26, 1, 43-61. doi: 10.1080/01441640500158496

Farag, S.; Weltevreden, J.; van Rietbergen, T.; Dijst, M.; van Oort, F. (2006): E-shopping in the Netherlands: does geography matter? In: Environment and Planning B 33, 1, 59-74. doi: https://doi.org/ $10.1068 / \mathrm{b} 31083$

Gebhardt, H. (2002): Neue Lebens- und Konsumstile, Veränderungen des aktionsräumlichen Verhaltens und Konsequenzen für das zentralörtliche System. In: Blotevogel, H. H. (Hrsg.): Fortentwicklung des Zentrale-Orte-Konzepts. Hannover, 91-103. = Forschungs- und Sitzungsberichte der ARL 217.

Gefen, D.; Karahanna, E.; Straub, D. A. (2003): Trust and TAM in Online Shopping: An Integrated Model. In: Management Information Systems Quarterly 27, 1, 51-90.

GfK Geo Marketing (2015): Ecommerce: Wachstum ohne Grenzen? Online-Anteile der Sortimente - heute und morgen. Bruchsal. http://www.gfk-geomarketing.de/white_paper_ecommerce.html (12.01.2018).
Girard, T.; Korgaonkar, P.; Silverblatt, R. (2003): Relationship of Type of Product, Shopping Orientations, and Demographics with Preference for Shopping on the Internet. In: Journal of Business and Psychology 18, 1, 101-120. doi: 10.1023/A:1025087021768

Gong, W.; Stump, R. L.; Maddox, L. M. (2013): Factors influencing consumers' online shopping in China. In: Journal of Asia Business Studies 7, 3, 214-230. doi: 10.1108/JABS-02-2013-0006

HDE - Handelsverband Deutschland (2017): Handel digital. Online-Monitor 2017. Berlin. https://www.einzelhandel.de/images/ presse/Pressekonferenz/2017/HDE_IFH_OnineMonitor_2017_2. pdf (12.01.2018).

Heinemann, G. (2014): Der neue Online-Handel. Geschäftsmodell und Kanalexzellenz im E-Commerce. Wiesbaden.

Heinemann, G. (2017): Die Neuerfindung des stationären Einzelhandels: Kundenzentralität und ultimative Usability für Stadt und Handel der Zukunft. Wiesbaden.

Hernández, B.; Jiménez, J.; Martín, M. J. (2011): Age, gender and income: do they really moderate online shopping behaviour? In: Online Information Review 35, 1, 113-133. doi: $10.1108 / 14684521111113614$

Hover, M. (2016): Die Auswirkungen des Online-Handels auf die Immobiliennachfrage in den Innenstädten. Masterarbeit, RWTH Aachen.

IFH - Institut für Handelsforschung (2016): Branchenreport: Onlinehandel. Köln.

Jürgens, U. (2014): Forschungsfragen zur Nahversorgung. In: Jürgens, U. (Hrsg.): Entwicklungen und Perspektiven von Nahversorgung im Lebensmitteleinzelhandel. Kiel, 1-6. = Kieler Arbeitspapiere zur Landeskunde und Raumordnung 54.

Kacen, J. J.; Hess, J. D.; Wei-Yu, K. C. (2013): Bricks or Clicks? Consumer Attitudes toward Traditional Stores and Online Stores. In: Global Economics and Management Review 18, 1, 12-21. doi: 10.1016/S2340-1540(13)70003-3

Kläsgen, M. (2017): Amazon kauft Whole Foods - Nein, die Läden in der Stadt sind nicht todgeweiht. In: Süddeutsche Zeitung vom 19. Juni 2017. http://www.sueddeutsche.de/wirtschaft/ amazon-kauft-whole-foods-nein-die-laeden-in-der-stadt-sindnicht-todgeweiht-1.3549183 (17.01.2018).

KPMG (2016): Trends im Handel 2025. Erfolgreich in Zeiten von Omni-Business. o. O.

Kulke, E. (2017): Wirtschaftsgeographie. Paderborn.

Langsenkamp, M. (2014): Der Einfluss von Online-Shopping auf den stationären städtischen Einzelhandel. Auswirkungen, Zusammenhänge und ökonomische Effekte untersucht am Beispiel des Kreises Paderborn. In: Geographische Kommission für Westfalen (Hrsg.): Die Rolle des Einzelhandels für die Stadtentwicklung. Die Bedeutung der Wochenmärkte und die Auswirkungen des Online-Handels. Münster, 120-266. = Westfälische Geographische Studien 59.

Lian, J.-W.; Yen, D. C. (2014): Online shopping drivers and barriers for older adults: Age and gender differences. In: Computers in Human Behavior 37, 133-143. doi: 10.1016/j.chb.2014.04.028

Mensing, M.; Neiberger, C. (2016): Mapping E-Commerce - regionale Unterschiede im Online-Einkaufsverhalten deutscher Verbraucher. In: Franz, M.; Gersch, I. (Hrsg.): Online-Handel ist Wandel. Mannheim, 109-132. = Geographische Handelsforschung 24.

Nerlich, M. R.; Schiffner, F.; Vogt, W. (2010): Einfluss des Internets auf das Informations-, Einkaufs- und Verkehrsverhalten. In: Lenz, B.; Kulke, E.; Nerlich, M.R.; Rauh, J.; Vogt, W. (Hrsg.): Produktion - Distribution - Konsum. Berlin, 75-115.

Otte, G. (2005): Entwicklung und Test einer integrativen Typologie der Lebensführung für die Bundesrepublik Deutschland. In: Zeitschrift für Soziologie 34, 6, 442-467. doi: 10.1515/zfsoz-20050606

Passyn, K. A.; Diriker, M.; Settle, R. B. (2011): Images Of Online Versus Store Shopping: Have The Attitudes Of Men And Wom- 
en, Young And Old Really Changed? In: Journal of Business and Economics Research 9, 1, 99-110. doi: 10.19030/jber.v9i1.946

Pesch, F.; Schenk, M.; Sperle, T. (2003): Bedeutungswandel der Innenstädte und Nebenzentren in den Städten von Nordrhein-Westfalen. Expertise im Auftrag der Enquetekommission „Zukunft der Städte in NRW“ des nordrhein-westfälischen Landtags. Stuttgart.

Ren, F.; Kwan, M.-P. (2009): The Impact of Geographic Context on E-Shopping Behavior. In: Environment and Planning B 36, 2, 262-278. doi: https://doi.org/10.1068/b34014t

Sinai, T.; Waldfogel, J. (2004): Geography and the Internet: Is the Internet a Substitute or a Complement for Cities? In: Journal of Urban Economics 56, 1, 1-24. doi: 10.1016/j.jue.2004.04.001

Sorce, P.; Perotti, V.; Widrick, S. (2005): Attitude and age differences in online buying. In: International Journal of Retail and Distribution Management 33, 2, 122-132. doi: 10.1108/09590550510581458

Staatskanzlei Nordrhein-Westfalen (2013): Landesentwicklungsplan Nordrhein-Westfalen. Sachlicher Teilplan Großflächiger Einzelhandel. Düsseldorf.

Stepper, M. (2015): Einkaufsstandort Innenstadt. Qualifizierung innerstädtischer Einzelhandelslagen vor dem Hintergrund des zunehmenden Online-Shopping. Dissertation, Technische Universität Kaiserslautern.

Swinyard, W. R.; Smith, S. M. (2003): Why people (don't) shop online: A lifestyle study of the internet consumer. In: Psychology and Marketing 20, 7, 567-597. doi: 10.1002/mar.10087
Teller, C.; Elms, J. (2010): Managing the attractiveness of evolved and created retail agglomerations formats. In: Marketing Intelligence \& Planning 28, 1, 25-45. doi: 10.1108/02634501011014598

Teller, C.; Reutterer, T. (2008): The evolving concept of retail attractiveness: What makes retail agglomerations attractive when customers shop at them? In: Journal of Retailing and Consumer Services $15,3,127-143$. doi: 10.1016/j.jretconser.2007.03.003

Weltevreden, J.; van Rietbergen, T. (2009): The Implications of E-Shopping for in-Store Shopping at Various Shopping Locations in the Netherlands. In: Environment and Planning B 36, 2, 279299. doi: 10.1068/b34011t

Wu, S.-I. (2003): The relationship between consumer characteristics and attitude toward online shopping. In: Marketing Intelligence and Planning 21, 1, 37-44. doi: 10.1108/02634500310458135

Zacharakis, Z. (2017): Lokaso - Guten Tag, hier kommt Ihre Wurst. In: Die Zeit vom 20. Juni 2017. http://www.zeit.de/wirtschaft/ unternehmen/2017-05/lokaso-e-commerce-local-commercesiegen (17.01.2018).

ZIA - Zentraler Immobilienausschuss (2015): Wandel im Handel. ZIA-Position zur Zukunft des stationären Einzelhandels. Berlin. http://www.zia-deutschland.de/fileadmin/Redaktion/Positionen/ PDF/ZIA_Position_Wandel_im_Handel.pdf (08.01.2018).

\section{Affiliations}

\section{Claus-C. Wiegandt ${ }^{7}$ Sabine Baumgart ${ }^{1}$ - Nina Hangebruch ${ }^{1} \cdot$ Linus Holtermann $^{2}$. Christian Krajewski $\cdot$ Matthias Mensing ${ }^{4} \cdot$ Cordula Neiberger $^{4} \cdot$ Frank Osterhage $^{5} \cdot$ Verena Texier-Ast $^{6} \cdot$ Klaus Zehner $^{8} \cdot$ Björn Zucknik $^{2}$}

Prof. Dr. Sabine Baumgart

sabine.baumgart@tu-dortmund.de

Nina Hangebruch

nina.hangebruch@tu-dortmund.de

Linus Holtermann

linus.holtermann@rub.de

Dr. Christian Krajewski

krajewc@uni-muenster.de

Matthias Mensing

matthias.mensing@geo.rwth-aachen.de

Prof. Dr. Cordula Neiberger

neiberger@geo.rwth-aachen.de

Frank Osterhage

frank.osterhage@ils-forschung.de

Verena Texier-Ast

texieras@uni-muenster.de

Prof. Dr. Klaus Zehner

k.zehner@uni-koeln.de
Björn Zucknik

bjoern.zucknik@rub.de

1 Fakultät Raumplanung, Technische Universität Dortmund, August-Schmidt-Straße 10, 44221 Dortmund, Deutschland

2 Geographisches Institut, Ruhr-Universität Bochum, Universitätsstraße 104, 44799 Bochum, Deutschland

3 Institut für Geographie, Westfälische Wilhelms-Universität Münster, Heisenbergstraße 2, 48149 Münster, Deutschland

4 Geographisches Institut, RWTH Aachen, Templergraben 55, 52056 Aachen, Deutschland

5 ILS - Institut für Landes- und Stadtentwicklungsforschung, Brüderweg 22-24, 44135 Dortmund, Deutschland

6 Institut für Geographie, Westfälische Wilhelms-Universität Münster, Heisenbergstraße 2, 48149 Münster, Deutschland

7 Geographisches Institut, Rheinische Friedrich-WilhelmsUniversität Bonn, Meckenheimer Allee 166, 53115 Bonn, Deutschland

8 Geographisches Institut, Universität zu Köln, Otto-FischerStraße 4, 50674 Köln, Deutschland 